\title{
COULD THE COMPACT REMNANT OF SN 1987A BE A QUARK STAR?
}

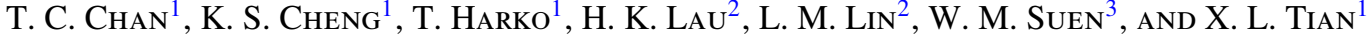 \\ ${ }^{1}$ Department of Physics and Center for Theoretical and Computational Physics, The University of Hong Kong, Hong Kong, People's Republic of China \\ ${ }^{2}$ Department of Physics and Institute of Theoretical Physics, The Chinese University of Hong Kong, Hong Kong, People's Republic of China \\ ${ }^{3}$ McDonnell Center for the Space Sciences, Department of Physics, Washington University, St. Louis, USA \\ Received 2008 September 20; accepted 2009 January 14; published 2009 April 1
}

\begin{abstract}
The standard model for Type II supernovae explosions, confirmed by the detection of neutrinos emitted during the supernova explosion, predicts the formation of a compact object, usually assumed to be a neutron star. However, the lack of detection of a neutron star or pulsar formed in the SN 1987A still remains an unsolved mystery. In this paper, we suggest that the newly formed neutron star at the center of SN 1987A may undergo a phase transition after the neutrino trapping timescale $(\sim 10 \mathrm{~s})$. Consequently the compact remnant of SN 1987A may be a strange quark star, which has a softer equation of state than that of neutron star matter. Such a phase transition can induce stellar collapse and result in large amplitude stellar oscillations. We use a three-dimensional Newtonian hydrodynamic code to study the time evolution of the temperature and density at the neutrinosphere. Extremely intense pulsating neutrino fluxes, with submillisecond period and with neutrino energy (greater than $30 \mathrm{MeV}$ ), can be emitted because the oscillations of the temperature and density are out of phase almost $180^{\circ}$. If this is true we predict that the current X-ray emission from the compact remnant of SN 1987A will be lower than $10^{34} \mathrm{erg} \mathrm{s}^{-1}$, and it should be a thermal bremsstrahlung spectrum for a bare strange star with a surface temperature of around $\sim 10^{7} \mathrm{~K}$.
\end{abstract}

Key words: dense matter - neutrinos - stars: oscillations - supernovae: individual (1987A)

\section{INTRODUCTION}

Supernova 1987A, the brightest supernova seen in modern times, was a milestone in astronomy and fundamental physics. The detection of neutrinos from SN 1987A (Bionta et al. 1987; Hirata et al. 1987) confirmed the basic predictions of the physical mechanisms for Type II supernovae. The explosion is triggered by the collapse of massive stars. The core collapse of the massive star (with mass greater than $10 M_{\odot}$ ) is due to electron capture and photodisintegration, and it is halted when the center density exceeds the nuclear matter density. As the stellar core collapses, the gravitational energy is released through the emission of neutrinos. A small fraction of these neutrinos is absorbed, and the heating of the neutrinos drives the supernova explosion.

The supernova SN 1987A was observed in every band of the electromagnetic spectrum, from radio to gamma rays, and hence it has a well-measured bolometric light curve. By using both the bolometric light curve and the spectral evolution of its $\mathrm{H} \alpha$ line, the hydrodynamic and time-dependent atmosphere models are consistent with a presupernova radius of $35 \pm 5 R_{\odot}$, an ejecta mass of $18 \pm 1.5 M_{\odot}$, an explosion energy of $(1.50 \pm 0.12) \times$ $10^{51} \mathrm{erg}$, and a radioactive ${ }^{56} \mathrm{Ni}$ mass of $0.0765 M_{\odot}$ (Utrobin 2007). The inferred energy $\left(\sim 3 \times 10^{53} \mathrm{erg}\right)$, temperature $(\sim 10 \mathrm{MeV})$, and decay time $(\sim 4 \mathrm{~s})$ of the first neutrino burst were not inconsistent with what would be expected from the production of a neutron star through core collapse (Hirata et al. 1987). Currently, about 35 plausible, or at least possible, associations of pulsars with supernova remnants are known, and these are all relatively young pulsars, mostly with $\tau_{p}=P / 2 \dot{P}<$ $10^{5}$ years, where $\dot{P}$ is the first derivative of the pulsar period $P$ (Manchester 2007). Almost immediately after the observation of SN 1987A, optical attempts were made to identify the compact remnant of the explosion (see Manchester 2007 for a recent review of the optical and X-ray observations on the supernova). Optical searches were done by Percival et al. (1995), using the High-Speed Photometer System on the Hubble Space Telescope, and by Manchester \& Peterson (1996), using the
$3.9 \mathrm{~m}$ Anglo-Australian Telescope, respectively. No significant pulsations were observed in the period range of $0.2 \mathrm{~ms}$ to $10 \mathrm{~s}$, with an upper limit for the pulsed emission equivalent to a $V$ magnitude of about 25. However, most pulsars are detected at radio wavelengths. An extensive search was carried out at the Parkes $64 \mathrm{~m}$ radio telescope in 2006, at frequencies between $1390 \mathrm{MHz}$ and $8370 \mathrm{MHz}$, respectively (Manchester 2007). No significant candidate with a signal-to-noise ratio greater than 9.0 was observed, and there is no observational evidence for the presence of a central point (or near point) source at any wavelength (Park et al. 2004; Graves et al. 2005; Shtykovskiy et al. 2005). The limit on the luminosity of any point source at the center of SN 1987A is of the order of $10^{33} \mathrm{erg} \mathrm{s}^{-1}-10^{34} \mathrm{erg} \mathrm{s}^{-1}$ (Manchester 2007).

Therefore, the question of why no compact object is observed at the center of the SN 1987A explosion is a rather intriguing one. Several explanations have been advanced to explain the lack of a neutron star/pulsar. The most obvious one is that the pulsar may not be beamed toward us (Manchester 2007). The beaming fraction (fraction of the celestial sphere swept over by the beam as the star rotates) is not very well determined, but in the radio band a beaming fraction of $20 \%$ is usually assumed, and for young pulsars it could be even larger (Manchester 2007). However, even if the pulsar were not directed along our line of sight, its radiation would heat the surrounding supernova remnant, and add to its bolometric luminosity. At present, all of the luminosity of SN 1987A can be accounted for by a radioactive decay model consistent with the production of $0.075 M_{\odot}$ of ${ }^{56} \mathrm{Ni}$ (Fryer et al. 1999; Utrobin 2007). It also would be possible that the pulsar magnetic field develops in a few decades, and in this case a rapidly spinning neutron star would still be undetectable (Manchester 2007). However, most of the current models assume that the magnetic field is either frozen in the star, or it is generated during the collapse by the dynamo process (Bonanno et al. 2005). Another possibility is that the neutron star further collapsed into a black hole, whose accretion luminosity is below the observational limits (Bethe \& 
Brown 1995). Although the duration of the neutrino burst of SN 1987A precludes further collapse in the first $20 \mathrm{~s}$, the neutron star could have collapsed eventually into a black hole either because of accretion of a sufficient fallback mass, or due to some changes in the equation of state (EOS) of the dense matter (Fryer et al. 1999). The analysis of the collapse of the initially formed neutron star in SN 1987A puts severe constraints on the EOS of the dense matter. However, to explain the collapse of a low-mass neutron star an exotic EOS of the nuclear matter is needed, like, for example, an EOS softened by pion condensation (Bethe \& Brown 1995), which would contradict other observations on supernova remnants. Initially, it is likely that neutron stars formed in Type II supernova explosions are obscured by the late-time fallback. Much of this fallback is quickly accreted via neutrino cooling, but some material may remain on the neutron star, forming an atmosphere that slowly accretes through photon emission. If the neutron star has either a low magnetic field, or a low rotational spin frequency, then the neutron star remnant of SN 1987A cannot be seen (Fryer et al. 1999).

When studying the neutrino data from the SN 1987A explosion, a number of unexpected features have been found (Hirata et al. 1987; Bionta et al. 1987; Costantini et al. 2004, 2007). For example, the angular distribution of the two events, one seen at the Kamiokande-II (KII) (Hirata et al. 1987) and the other at Irvine-Michigan-Brookhaven (IMB) (Bionta et al. 1987) are more forward directed than expected. The average cosines of the polar angles for these two events are $\left\langle\cos \theta^{\mathrm{KII}}\right\rangle \approx 0.3$ and $\left\langle\cos \theta^{\mathrm{IMB}}\right\rangle \approx 0.5$. Moreover, the energy distribution of these two detectors is not in perfect agreement. The mean energy of the neutrinos detected at KII is around half of the energy of those detected at IMB, with a mean energy of $\left\langle E^{\mathrm{IMB}}\right\rangle \approx 30 \mathrm{MeV}$. The time distribution of the two events is also very different; on average the neutrinos detected by IMB came $\sim 5 \mathrm{~s}$ after those detected by KII (see Alekseev et al. 1988 for a summary of neutrino arrival time for various detectors). When fitted with thermal spectra, the two independent detections do not seem to agree with either each other or typical theoretical expectations. Using parameter-free inferential statistical methods, it can be shown that the combined KII and IMB data can be best explained by a spectral shape that is enhanced both at the peak and the tail of the spectrum, and depressed in between, as compared to the Fermi-Dirac spectrum (Yüksel \& Beacom 2007). Since these methods make no a priori assumptions and do not rely on parameter estimation, they allow for more efficient processing of small data samples. While the supernova neutrino spectra are expected to be quasi-thermal, modifications due to nonstandard effects, such as neutrino mixing among various flavors, neutrino decay, neutrino-neutrino interactions and/or any novel mechanism due to unknown physics, may produce a time-integrated spectrum that deviates significantly from a quasi-thermal shape (Yüksel \& Beacom 2007).

It is the purpose of the present paper to propose some explanations for the lack of observational evidence for the presence of a point source at the center of SN1987 A, as well as the apparent discrepancy between the two neutrino detections.

Since Witten (1984), following early proposals by Itoh (1970) and Bodmer (1971), suggested that strange quark matter, consisting of $u-, d$ - and $s$-quarks, is energetically the most favorable state of the matter, the problem of the existence of strange quark stars has been intensively investigated in the physical and astrophysical literature. The possibility that some compact objects could be strange stars remains an interesting and intriguing, but still open, question. Witten (1984) also proposed two methods of formation of strange matter: the quark-hadron phase transition in the early universe and conversion of neutron stars into strange ones at ultrahigh densities. In the theories of strong interaction quark bag models suppose that breaking of a physical vacuum takes place inside hadrons. As a result vacuum energy densities inside and outside a hadron become essentially different, and the vacuum pressure on the bag wall equilibrates the pressure of quarks, thus stabilizing the system. If the hypothesis of the quark matter is true, then some of the neutron stars could actually be strange stars, built entirely of strange matter (Haensel et al. 1986; Alcock et al. 1986; Alcock \& Olinto 1989). However, there are general arguments against the existence of strange stars, e.g., Caldwell \& Friedmann (1991). For a general review of strange star properties and the physics of phase transitions see Cheng et al. (1998a) and Haensel et al. (2007).

A possibility for the formation of the quark stars is that some neutron stars in low-mass X-ray binaries can accrete sufficient mass to undergo a phase transition to become strange stars (Cheng \& Dai 1996). This mechanism has also been proposed as a source of radiation emission for cosmological gamma-ray bursts, soft gamma-ray repeaters or other astrophysical objects (Cheng \& Dai 1998; Cheng et al. 1998b; Paczynski \& Haensel 2005). Some basic properties of strange stars, such as mass, radius, cooling, collapse and surface radiation, have also been studied (Christiansen et al. 2000; Cheng \& Harko 2003; Harko \& Cheng 2000, 2002; Zdunik et al. 2001; Gondek-Rosinska et al. 2003; Bejger \& Haensel 2004; Zdunik et al. 2006). Quark stars are expected to form during the collapse of the core of a massive star after the supernova explosion, as a result of a first- or secondorder phase transition, resulting in deconfined quark matter (Takahara \& Sato 1985a, 1985b; Gentile et al. 1993; Dai et al. 1995). The proto-neutron star core, or the neutron star core, is a favorable environment for the conversion of ordinary matter to strange quark matter (Chen et al. 2007).

This paper is organized as follows. In Section 2, we discuss various possible phase-transition processes in a neutron star, and estimate the characteristic timescale for the phase transition. We suggest that the phase transition from neutron star matter to strange matter in the core of a neutron star may be more favorable. In Section 3, we present the numerical simulation results of a phase-induced collapse neutron star. In particular, we show why high-intensity and high-energy neutrinos can be emitted after the phase transition. In Section 4, we summarize the energy dissipation processes of bare strange stars and their cooling. In Section 5, we give a brief discussion about how the phase transition may take place in the compact remnant of 1987A, and present final remarks.

\section{PHASE TRANSITIONS IN HIGH-DENSITY NEUTRON MATTER}

During the formation and evolution of neutron stars new states of matter may form inside the stars as a result of a phase transition, which may be triggered by the accretion of matter, pulsar spindown, and collapse of the core of a proto-neutron star in supernova explosions, etc. In the following, we shall restrict our discussion only to phase transitions of the first order. As a general physical model, we will discuss the nucleation of a phase $B$ in the metastable phase $A$. The nucleation is concerned with fluctuations of parameters, such as the local density or the number of particles in a metastable drop of phase $B$ which triggers the phase transition (Landau \& Lifshitz 1980). 
There are two extreme cases of the nucleation theory. In the classical regime, the temperature is assumed to be sufficiently high to trigger the phase transition by thermal fluctuation. In the quantum regime, below a characteristic temperature, thermal fluctuations are negligible as compared to the quantum ones. Hence, quantum fluctuations can initiate a phase transition via the quantum tunneling effect. The relevant thermodynamic potential for the description of phase transitions is the Gibbs free energy (Haensel \& Schaeffer 1982). In the following, we denote by $\Delta F$ the excess free energy of a critical droplet.

The probability of a local formation of a droplet of phase $B$ via a fluctuation decreases very strongly with the increase of the number of baryons $A_{\text {drop }}$ in the droplet. For small $A_{\text {drop }}$, however, the positive contribution of the surface energy to $\Delta F$ prevails over the gain in the bulk binding, $\Delta F\left(A_{\text {drop }}\right)>0$, which makes the droplet unstable with respect to the reconversion to phase $A$. However, at some value $A_{\text {drop }}=A_{\text {crit }}$, the energy excess due to the droplet formation vanishes, $\Delta F\left(A_{\text {crit }}\right)=0$. Therefore the droplets with $A_{\text {drop }}>A_{\text {crit }}$ grow spontaneously, destabilizing the metastable phase $A$ and inducing the phase transition (Harko et al. 2004).

By using the thermodynamical formalism of nucleation (Landau \& Lifshitz 1980; Haensel \& Schaeffer 1982; Haensel et al. 2007), one can study the nucleation of exotic phases (pion condensate, kaon condensate, and quark matter) in high-density neutron matter. In the following, we will briefly review each of these processes.

\subsection{Meson Condensation}

The nucleation of the pion condensate in the neutron star core and its astrophysical implications were studied by Haensel \& Schaeffer (1982), Haensel \& Proszynski (1982), and Muto \& Tatsumi (1990), respectively, where a theory of metastability of dense neutron matter with respect to the first-order phase transition to a pion-condensed state was also analyzed. The description of the condensation process is based on the idea of the nucleation of the pion-condensed phase in the metastable normal matter, through the appearance of spontaneously growing droplets of the new phase. Different paths leading from the false to the true ground state of dense neutron matter have been considered in Haensel \& Schaeffer (1982).

The calculations performed for realistic models of cold neutron matter yield an interval of metastability (on the timescale of the age of the universe), which is as large as half of that between the baryon density $\rho_{N}$, where the metastability starts, and $\rho_{\text {crit }}$, where the potential barrier (without surface effects) between the true and the false ground state vanishes (Haensel \& Schaeffer 1982). In the case of pion condensation in hot neutron matter $(T \sim 5 \mathrm{MeV})$, the region of metastability (on the timescale of the gravitational collapse of a massive star) is much narrower than in cold neutron matter. Consequently, additional energy release and entropy generation from the first-order phase transition in hot supercompressed matter are negligible. The value of the $A_{\text {crit }}$ strongly decreases with the growth of the overcompression $\Delta P_{\text {over }}=P-P_{0}$. Thus, the lifetime of an overcompressed state decreases rapidly with increasing $\Delta P_{\text {over }}$ (Haensel \& Schaeffer 1982; Haensel et al. 2007). For the case of the pion condensation the surface tension can be approximated by the expression derived by Baym et al. (1971), with the nucleus replaced by a pion-condensed droplet, and the neutron gas replaced by the ordinary neutron star matter. Then, one can obtain the condition for the pion condensate in the neutron star core during a time interval equal to the present age of the universe,
$t_{H}=1.5 \times 10^{10}$ years. Depending on the employed model, the required overcompression is $\Delta P_{\text {over }} / P_{0}=0.02$ or $\Delta P_{\text {over }} / P_{0}=$ 0.05 (Muto \& Tatsumi 1990; Haensel et al. 2007).

Under typical conditions in the neutron star core $\left(T \leqslant 10^{9} \mathrm{~K}\right)$, the nucleation proceeds via quantum tunneling through the energy barrier. At much higher temperatures, thermal effects increase the nucleation rate through thermally excited droplet states. On the other hand, the growth of the temperature increases $P_{\text {crit }}$. For a newly born neutron star with $T \geqslant 10^{10} \mathrm{~K}$, the nucleation proceeds in the classical (thermal) regime (Haensel et al. 2007). Due to the softening of the EOS, pion condensation could lead to a significant decrease of the maximum mass and moment of inertia allowable for neutron star models, constructed using such an EOS (Haensel \& Proszynski 1982).

The formation of a droplet of kaon condensate in a neutron star core is connected with the production of strangeness, and nucleation should involve the weak-interaction process. The transition from npe-type nuclear matter (consisting of neutrons, protons, and electrons) to matter containing strangeness, using a Walecka-type model, predicting a first-order kaon-condensate phase transition, was studied in Norsen (2002). The free energy of the droplets of the kaon-condensed matter, as well as the density, temperature, and the neutrino fraction, was obtained. The surface tension of the interface between the normal and condensed kaon phase was calculated by Christiansen et al. (2000), using the nonuniform relativistic mean field model. In the approximation in which only linear terms in the curvature of the surface are kept, the surface contribution to the thermodynamic potential of a spherical droplet is $\sigma=\sigma_{S}+2 \sigma_{c} / R_{\text {drop }}$, where $\sigma_{S}$ is the surface tension and $\sigma_{c}$ is the curvature coefficient. For a small admixture of kaon-condensed droplets in nucleon matter, $\sigma_{S}=30 \mathrm{MeV}$ (Christiansen et al. 2000).

The case of kaon condensation is drastically different from the case of the deconfinement transition, since here there is no intermediate zero-strangeness state, which might allow for fast nucleation followed by a slow but smooth growth of the strangeness containing fields. Instead, the thermal fluctuations responsible for nucleation events must directly involve weakinteraction processes which produce kaons. Weak-interaction processes producing strangeness via the reaction $e+N \rightarrow$ $v_{e}+K^{-}+N$ (where an additional nucleon $N$ is needed for momentum conservation) and $n \rightarrow p+K^{-}$are too slow to create a critical droplet of kaon condensate from a density fluctuation during the fluctuation lifetime (Norsen 2002). Strangeness can be produced at a reasonable rate from thermal kaon-antikaon $\left(K^{-} K^{+}\right)$pairs, but this mechanism can operate only at extremely high temperatures typical for proto-neutron stars. Generally, a proto-neutron star cools so rapidly that kaon condensate has no time to nucleate (Haensel et al. 2007). However, as soon as $\mu_{e}>\omega_{K^{-}}^{0}$, where $\omega_{K^{-}}^{0}$ is the minimum energy of a single zero momentum kaon in dense matter, spontaneous formation of kaons is possible (Haensel et al. 2007). The kinetics of kaon condensation was studied by Muto et al. (1997, 2000a, 2000 b). Kaon potentials $U_{K}$ with values only a little below $U_{K}=-120 \mathrm{MeV}$ would not be compatible with the mass of the Hulse-Taylor pulsar, because of a mechanical instability that is initiated by the central densities for which the pressure remains constant, and the necessary condition for stability $d M / d \rho_{c}>0$ is not satisfied (Glendenning 2000). The kaon condensation process in neutron stars in the framework of the Zimanyi-Moszkowski model in relativistic mean field theory was considered in Dai \& Cheng (1997). Even though hyperons which may increase the critical condensation density are not 
included, kaon condensation may not occur in stable neutron stars for these classes of mean field theories. The existence of the antikaon condensation phase in neutron stars in the frameworks of the Glendenning-Moszkowski and Zimanyi-Moszkowski models was re-analyzed in Wang et al. (2007). The results of this analysis show that in the very massive $\left(M=2 \pm 0.2 M_{\odot}\right)$, and high-redshift neutron stars, there are still some stiff enough EOSs of neutron matter, so that the pure antikaon condensation phase and the mixed phase of normal baryons and antikaon condensation can still exist. The mass of the neutron star inferred from the neutrino flux of SN 1987A should be of the order of 1.5-1.7 $M_{\odot}$, which suggests that the kaon condensate may not occur in the compact object of SN 1987A.

\subsection{Quark Deconfinement}

When quarks seeds are formed in the core of a neutron star, they will propagate through the entire star, and convert it to the new phase. A phase transition occurs between the hadronic and quark phases when the pressures and the chemical potentials in the two phases are equal, $P_{h}=P_{q}, \mu_{h}=\mu_{q}$, where $P_{h}, \mu_{h}$ and $P_{q}, \mu_{q}$ are the pressures and the chemical potentials in the hadron and quark phases, respectively. If the transition pressure is less than that existing in the supernova core the transition can occur.

The change from the metastable neutron matter phase to the stable quark phase occurs as the result of fluctuations in a homogeneous medium, formed of neutrons, in which small quantities of the quark phase (called bubbles or nuclei) are randomly generated. Since the process of creation of an interface is energetically unfavorable, it follows that when a quark nucleus is below a certain size, it is unstable and disappears again. Surface effects disfavor the survival of small bubbles below the radius $R_{c}$ (called critical size-nuclei of this size are called critical nuclei or bubbles), which is nothing but the value that extremizes the thermodynamical work $W$ necessary to create the bubbles (Alcock \& Olinto 1989; Harko et al. 2004). Only nuclei whose size $r$ is above the value $R_{c}$ are stable, and can survive (Landau \& Lifshitz 1980). The nuclei are assumed to be macroscopic objects containing a large number of particles (quarks).

Following the phase transition to two-flavor quark matter, the two-flavor quark matter will convert into three-flavor quark matter through the reactions $u+e^{-} \leftrightarrow d+v_{e}, u+e^{-} \leftrightarrow s+v_{e}$, and $u+d \leftrightarrow u+s$, respectively (Dai et al. 1995).

Once the quark phase is formed inside the neutron star, it will propagate throughout the entire star. The physical mechanisms of the transition from neutron matter to quark matter in an astrophysical background have been studied within several models. The first is due to Olinto (1987), who used a nonrelativistic diffusion model. As such, this is a slow combustion model, with the burning front propagating at a speed of approximately $10 \mathrm{~m} \mathrm{~s}^{-1}$. This is determined primarily by the rate at which one of the down quarks inside the neutrons is converted, through weak decay, to a strange quark: $d+u \rightarrow s+u$. The second method of describing the conversion process was first suggested by Horvath \& Benvenuto (1988), and analyzed in detail by Lugones et al. (1994) and Lugones \& Benvenuto (1995), who modeled the conversion as a detonation. In this case, the conversion rate is several orders of magnitude faster than that predicted by the slow combustion model. This model is based on relativistic shock waves and combustion theory. But regardless of the way in which the transformation occurs, an initial seed of quark matter is needed to start the process.
The problem of the combustion of neutron matter is closely related to that of shock waves. Let us assume that we have an unburnt fluid which converts (by means of a certain reaction) to a burnt fluid. The combustion process must be exothermic if it propagates spontaneously to other regions of the fluid. The condition for spontaneous propagation to other regions of the fluid is $E_{\text {burnt }}(P, X)<E_{\text {unburnt }}(P, X)$, where $E_{\text {burnt }}(P, X)$ and $E_{\text {unburnt }}(P, X)$ are the energy densities of the respective fluids, both evaluated at the same thermodynamic state. In the case of the transition from nuclear to quark matter this condition can be reformulated as $E_{\text {nucl }}-3 P_{\text {nucl }}>4 B$ (Lugones et al. 1994), where $E_{\text {nucl }}$ and $P_{\text {nucl }}$ correspond to nuclear matter. If this condition is not fulfilled, the combustion is no longer exothermic, and so it is not possible. At low enough densities, soft EOS verifies $E_{\text {nucl }} \gg P_{\text {nucl }}$, and $E_{\text {nucl }} \approx m_{n} n_{B} c^{2}$. Hence there exists an absolute lower limit for the combustion to be possible, given by $n_{B} \approx 4 B / m_{n} c^{2}=0.25\left[B /(145 \mathrm{MeV})^{4}\right] \mathrm{fm}^{-3}$ (Lugones et al. 1994), corresponding to a transition density $\rho_{\text {tr }}$ of the order of $\rho_{\text {tr }} \approx 4.2 \times 10^{14}\left[B /(145 \mathrm{MeV})^{4}\right] \mathrm{g} \mathrm{cm}^{-3}$.

However, it is important to point out that efficient burning of the nuclear matter can take place only when the nuclear matter density is sufficiently high, and in order to convert the entire neutron star to a quark star a higher density than the minimum one given by $n_{B}$ is required, and there is always a range of densities at which the transition occurs. The density for the deconfinement of baryonic matter with a moderately stiff (or stiff) EOS to two-flavor quark matter is near $8 \rho_{\text {nuc }}$. For a soft EOS, the deconfinement density may be lower.

Generally, the flow behind the detonation is sonic. Therefore the typical timescale for the transition is $\tau_{\mathrm{tr}}=R / c_{s}$, where $R$ is the radius of the neutron star and $c_{s}$ is the speed of sound. A simple phenomenological model for the evolution of the quark phase can be obtained by assuming $d r / d t=\left(r-R_{c}\right) / \tau_{\text {tr }}$ (Harko et al. 2004), which gives for the transition timescale $T_{\text {tr }}$ from a microscopic quark nugget to a quark matter distribution of a macroscopic size the expression

$$
T_{\text {tr }} \approx 10^{-4} N_{q}^{-1 / 3} R_{6} \ln \frac{R}{R_{q}} \mathrm{~s},
$$

where $R_{6}$ is the neutron star radius in units of $10^{6} \mathrm{~cm}, R_{q} \sim$ $300 R_{c}$ (Harko et al. 2004) is the initial size of the quark bubble, and $N_{q}$ is the number of quark seeds inside the core of the neutron star, which could be as large as $10^{48}$ (Iida \& Sato 1998). However, it was found that the conversion process in hadronic matter at $T=0$ always corresponds to a deflagration, and never to a detonation (Drago et al. 2007). Hydrodynamical instabilities can develop on the front, and a mixed phase of hadrons and quarks could form. Due to the formation of wrinkles, the conversion velocity can significantly increase, but this increase is not sufficient to transform the deflagration into a detonation. In general, one could assume that the conversion process can take place in two steps, with a first transition from hadrons to ungapped (or 2SC) quarks, followed by a second transition in which a color-flavor locked (CFL) phase is produced. The timescale for the first transition has been estimated by Drago et al. (2007), and it is of the order of $0.1-1 \mathrm{~s}$ for the first transition in the case of a laminar front, and is much more rapid if the hydrodynamical instabilities are taken into account. The second transition lasts only $10^{-3} \mathrm{~s}$, due to the formation of a convective layer. If the two processes take place one after the other, it is even possible that the formation of a diquark condensate could accelerate the conversion process by developing a convective layer inside the hadronic phase. Neutrino trapping can play an important 
role in the hadron-quark phase transition (Vidana et al. 2005). The quantum nucleation of a quark matter drop, and therefore the conversion of the hadronic matter to quark matter, is strongly inhibited at $T=0$ by the presence of neutrinos. However, in the high-temperature regime, the dominant nucleation mechanism is thermal nucleation, and not quantum tunneling (Haensel et al. 2007). In general, it takes a submillisecond time interval to convert the matter at the core of a neutron core into quark matter.

\section{NEUTRINO EMISSION FROM A PHASE-INDUCED COLLAPSE NEUTRON STAR}

The total energy emitted in the form of neutrinos in a supernova explosion can easily be estimated from qualitative considerations. The total gravitational energy that can be irradiated is $E_{b} \approx 3 G M^{2} / 5 R$, where $M$ and $R$ are the mass and the radius of the star, respectively. By using a mass of the order of $M=(1-2) M_{\odot}$, and a radius of $R=20 \mathrm{~km}\left(M_{\odot} / M\right)^{1 / 3}$ for the neutron star, one obtains $E_{b} \approx(1-5) \times 10^{53} \mathrm{erg}$. This amount of neutrino energy does not conflict with the observed data. However, it is difficult to understand why, in comparing with KII, IMB has detected a neutrino burst with a time delay $(\sim 5 \mathrm{~s})$ and with even higher average energy $(\sim 30 \mathrm{MeV})$. In the discussion section, we will argue that a phase transition may take place after the core temperature of the newly born neutron star is reduced. The characteristic timescale for the cooling of the core is the neutrino trapping time, which is of the order of several seconds. In this section, we use a three-dimensional Newtonian hydrodynamic code to simulate the neutrino emission from a phase-induced collapse of a neutron star.

\subsection{Description of the Numerical Code}

First, we briefly summarize the numerical code used to simulate the collapse of a neutron star induced by phase transition. The three-dimensional simulations are based on Newtonian hydrodynamics and gravity. The code has been used to study the gravitational wave emission from phase-induced collapse neutron stars (Lin et al. 2006). We refer the reader to Lin et al. (2006) for a detailed discussion of the numerical code.

The system of equations describing the nonviscous Newtonian fluid flow is given by

$$
\begin{gathered}
\frac{\partial \rho}{\partial t}+\nabla \cdot(\rho \mathbf{v})=0, \\
\frac{\partial}{\partial t}\left(\rho v_{i}\right)+\nabla \cdot\left(\rho v_{i} \mathbf{v}\right)+\frac{\partial P}{\partial x_{i}}=-\rho \frac{\partial \Phi}{\partial x_{i}}, \\
\frac{\partial \tau}{\partial t}+\nabla \cdot((\tau+P) \mathbf{v})=-\rho \mathbf{v} \cdot \nabla \Phi,
\end{gathered}
$$

where $\rho$ is the mass density of the fluid, $\mathbf{v}$ is the velocity with Cartesian components $v_{i}(i=1,2,3), P$ is the fluid pressure, $\Phi$ is the Newtonian potential, and $\tau$ is the total energy density, $\tau=\rho \epsilon+\rho \mathbf{v}^{2} / 2$, where $\epsilon$ is the internal energy per unit mass of the fluid. The Newtonian potential $\Phi$ is obtained by solving the Poisson equation, $\nabla^{2} \Phi=4 \pi G \rho$. The system is completed by specifying an EOS $P=P(\rho, \epsilon)$.

The above hydrodynamics Equations (2)-(4) can be rewritten in a flux conservative form, which can be solved numerically using quite standard high-resolution shock capturing (HRSC) schemes. An HRSC scheme has the ability to resolve discontinuities (e.g., shock waves) in the solution. It can also achieve high accuracy in regions where the fluid flow is smooth. In our work, we employ the so-called Roe's solver in the simulations (see Lin et al. 2006).

It is not known what the EOS is for the neutron star in the remnant of SN 1987A. We can try all possible existing realistic EOSs in our study. However, the main purpose of this paper is to demonstrate that the phase-induced collapse of a neutron star can emit extremely intense, pulsating and very high energy neutrinos. Therefore, instead of using a realistic EOS we will use a polytropic EOS for the initial neutron star. In Section 2, we have discussed various possible phase transitions for a newly born hot neutron star. Although both pion condensate and kaon condensate cannot be ruled out completely, there is no compelling observational evidence for the their existence. For simplicity, we will focus our study on the phase transition from neutron star to strange star in this paper. We use a mixed phase EOS to mimic a strange star covered by normal nuclear matter.

In the following, we argue that a newly born quark star should be described by a mixed phase EOS. In Section 2.2, it has been shown that quark seeds can be spontaneously generated inside the core of neutron star when the density exceeds the critical density (e.g., Iida \& Sato 1998). These quark seeds formed in the neutron star core can mix with the normal hadronic matter by Schwarzschild convection, and the length scale $\lambda_{c}$ for the convective motion is given by (Wilson \& Mayle 1988)

$$
\lambda_{c}=\left[\frac{k_{T} \eta}{g(-d \ln S / d r)}\right]^{1 / 4},
$$

where $S$ is the entropy per baryon in units of Boltzmann's constant, $g$ is the local gravitational acceleration in the core, $k_{T}=\lambda_{v} / 3$, where $\lambda_{v}$ is the neutrino mean free path, $\eta=$ $\lambda_{\nu} \rho_{\nu} / 3 \rho$, where $\rho_{\nu}$ is the neutrino energy density and $\rho$ is the matter density. With $\lambda_{v}=80 \mathrm{~cm}, \rho_{v} / \rho=0.1, g=8 \times 10^{13}$ $\mathrm{cm} \mathrm{s}^{-2}$, and $-d \ln S / d r=5 \times 10^{-6} \mathrm{~cm}^{-1}$ (Dai et al. 1995), one obtains $\lambda_{c}=3.5 \times 10^{3} \mathrm{~cm}$. The timescale of the mixing can be calculated from $\tau_{c}=2 \pi / \sqrt{g(-d \ln S / d r)}=0.3 \mathrm{~ms}$. Therefore, the convection will result in mixing of the neutron and quark phases in the large region of the star during the phasetransition process (Glendenning 2000).

The initial equilibrium neutron star before the phase is given by a polytropic EOS

$$
P=k_{0} \rho^{\Gamma_{0}},
$$

where $k_{0}$ and $\Gamma_{0}$ are constants. On the initial time slice, we also need to specify the specific internal energy $\epsilon$. For the polytropic EOS, the thermodynamically consistent $\epsilon$ is given by

$$
\epsilon=\frac{k_{0}}{\Gamma_{0}-1} \rho^{\Gamma_{0}-1} .
$$

Note that the pressure in Equation (6) can also be written as

$$
P=\left(\Gamma_{0}-1\right) \rho \epsilon .
$$

We assume that the phase transition take place at $t=0$, then we switch the compact object from a polytropic EOS to the EOS of a mixed phase quark star, which consists of two parts: (1) a mixed phase of quark and nuclear matter in the core at density higher than a certain critical value $\rho_{\text {tr }}$ (quark seeds can spontaneously produce everywhere when $\rho \geqslant \rho_{\text {tr }}$ ) and (2) a 
normal nuclear matter region extending from $\rho<\rho_{\text {tr }}$ to the surface of the star. Explicitly, the pressure is given by

$$
P= \begin{cases}\alpha P_{\mathrm{q}}+(1-\alpha) P_{\mathrm{n}} & \text { for } \rho>\rho_{\mathrm{tr}} \\ P_{\mathrm{n}} & \text { for } \rho \leqslant \rho_{\mathrm{tr}},\end{cases}
$$

where

$$
P_{\mathrm{q}}=\frac{1}{3}(\rho+\rho \epsilon-4 B)
$$

is the pressure contribution of the quark matter, and

$$
P_{\mathrm{n}}=\left(\Gamma_{n}-1\right) \rho \epsilon
$$

where $\Gamma_{n}$ is not necessarily equal to $\Gamma_{0}$, is that of the nuclear matter, and

$$
\alpha=\left\{\begin{array}{lc}
\left(\rho-\rho_{\mathrm{tr}}\right) /\left(\rho_{\mathrm{q}}-\rho_{\mathrm{tr}}\right) & \text { for } \rho_{\mathrm{tr}}<\rho<\rho_{\mathrm{q}} \\
1 & \text { for } \rho_{\mathrm{q}}<\rho
\end{array}\right.
$$

is defined to be the scale factor of the mixed phase (Lin et al. 2006). We should note that $P_{\mathrm{q}}$ is not in the usual form of MIT bag $P_{\mathrm{q}}=\frac{1}{3}\left(\rho_{\text {tot }}-4 B\right)$, where $\rho_{\text {tot }}$ is the (rest-frame) total energy density, and $B$ is the bag constant. It is because in Newtonian simulation we use the rest mass density $\rho$ and specific internal energy $\epsilon$ as fundamental variables in the hydrodynamics equations. The total energy density $\rho_{\text {tol }}$, which includes the rest mass contribution, is decomposed as $\rho_{\text {tot }}=\rho+\rho \epsilon$. We choose $\Gamma_{n}<\Gamma_{0}$ in our simulations to take into account the possibility that the nuclear matter may not be stable during the phase-transition process, and hence some quark seeds could appear inside the nuclear matter, or the convection mentioned in the early part of this section may mix some quark matter with the nuclear matter. In the presence of the quark seeds in the nuclear matter, the effective adiabatic index will be reduced. The possible values of $B^{1 / 4}$ range from $145 \mathrm{MeV}$ to $190 \mathrm{MeV}$ (DeGrand et al. 1975; Satz 1982; Steffens et al. 1995). For $\rho>\rho_{q}$, the quarks will be deconfined from the nucleons. The value of $\rho_{q}$ is model dependent; it could range from 4 to 8 $\rho_{\text {nuc }}$ (Cheng \& Dai 1998; Haensel 2003; Bombaci et al. 2004), where $\rho_{\text {nuc }}=2.8 \times 10^{14} \mathrm{gcm}^{-3}$ is the nuclear density.

In the simulations, we set $\Gamma_{0}=2, \Gamma_{n}=1.85, B^{1 / 4}=$ $160 \mathrm{MeV}$, and $\rho_{q}=9 \rho_{\text {nuc }}$. The phase-transition density $\rho_{\text {tr }} \approx$ $2.1 \rho_{\text {nuc }}$ is defined to be the point where $P_{\mathrm{q}}$ is zero initially. This value is approximately equal to the value estimated in Section 2.2. The total time span of each run is $\sim 5 \mathrm{~ms}$, the time step is $3.7 \times 10^{-4} \mathrm{~ms}$. The grid spacing is set to be $d x=$ $0.28 \mathrm{~km}$ and the outer boundary of the computational domain is at $27.5 \mathrm{~km}$, which is about twice the stellar radius of our models. During the numerical simulation, a low-density atmosphere is added outside the neutron star for numerical stability purposes. The density and temperature of the atmosphere are $3 \times 10^{9} \mathrm{gcm}^{-3}$ and $0.003 \mathrm{MeV}$, respectively.

Finally, we remark that although Equation (9) is not the exact situation of quark matter distribution inside the star, it allows us to simulate the phase-induced collapse and study the energy transport processes. The exact quark matter distribution can certainly affect the evolution of the star in detail and neutrino emission from the stellar surface quantitatively. However, since Equation (9) should still represent the qualitative quark matter distribution, the simulated features produced by Equation (9) should still be qualitatively correct.

\subsection{Neutrino Luminosity}

For a newborn neutron star, the internal temperature is so hot that neutrinos will be trapped inside the star for at least a few seconds (see Shapiro \& Teukolsky 1983 for a general review). However, neutrinos very near the surface of the star can still escape because the optical depth near the stellar surface is low. In fact we can define a radius $\left(R_{v}\right)$ called the neutrinosphere, where the optical depth of electron neutrinos is given by

$$
\tau_{\mathrm{eff}}=\int_{R_{v}}^{\infty} d r \kappa_{\mathrm{eff}}(r)=1,
$$

where the effective optical depth, $\tau_{\text {eff }}$, is defined as inverse mean free paths and the effective opacity, $\kappa_{\text {eff }}$, is given by (Janka 2001)

$$
\left\langle\kappa_{\mathrm{eff}}\right\rangle(r)=1.202 \times 10^{-7} \rho_{10}(r)\left(\frac{T_{\nu}(r)}{4 \mathrm{MeV}}\right)^{2} \frac{1}{\mathrm{~cm}},
$$

where $\rho_{10}(r)$ is the density in units of $10^{10} \mathrm{~g} \mathrm{~cm}^{-3}$ and $T_{v}(r)$ is the temperature at $r$, respectively. The electron neutrino luminosity emitted from the neutrinosphere is given by (Janka 1995; Balantekin \& Yuksel 2005)

$$
L_{v}=\frac{7}{16} \pi R_{v}^{2} a c T_{v}^{4} .
$$

In our simplified calculation, we also assume equal luminosities for the neutrino and antineutrino, hence the combined luminosity for a single neutrino flavor is

$$
\begin{aligned}
L_{v, \bar{v}} & =L_{v}+L_{\bar{v}} \\
& =\frac{7}{8} \pi R_{v}^{2} a c T_{v}^{4} .
\end{aligned}
$$

Since the neutrinosphere is very near the stellar surface, where the nuclear matter is described by an ideal gas EOS $P_{n}=\left(\Gamma_{n}-1\right) \rho \epsilon$ (see the previous section), we thus approximate the temperature by $T=2 m_{B} \epsilon / 3 k$, where $k$ is Boltzmann's constant and $m_{B}=1.67 \times 10^{-24} \mathrm{~g}$ is the baryon mass. With the simulated density and temperature profiles at a given time, we obtain the value of each $R_{v}$ from 0 to $3 \mathrm{~ms}$ with the trialand-error method. Since the neutrinosphere is a function of both temperature and density, which oscillates with a period $\sim 0.32 \mathrm{~ms}$, therefore it also oscillates with the same period. The temperatures and densities as a function of time are shown in Figures 1 and 2 for a neutron star with 1.55 and $1.75 M_{\odot}$, respectively.

From these two figures, it appears that the temperature oscillation and the density oscillation are almost close to $180^{\circ}$ out of phase. Since the entire system is oscillating, every quantity should oscillate with the same period. Also the equations governing the evolution of temperature and density are not identical; it is not surprising that they have a phase difference. However, the question is: why are they almost $180^{\circ}$ out of phase? This phenomenon can be explained qualitatively as follows. When the oscillatory cycle begins, matter is falling in until the end of the first half-cycle. However, once the matter is squeezed, the temperature is rising everywhere inside the star and the gradient of the temperature starts to drive the thermal energy outward. In fact, most of the thermal energy is generated in the core. Furthermore, the definition of the neutrinosphere is that the product of the temperature and density is a constant. Therefore, when the density is decreasing with time, but the temperature is increasing with time in the first half of the cycle, 

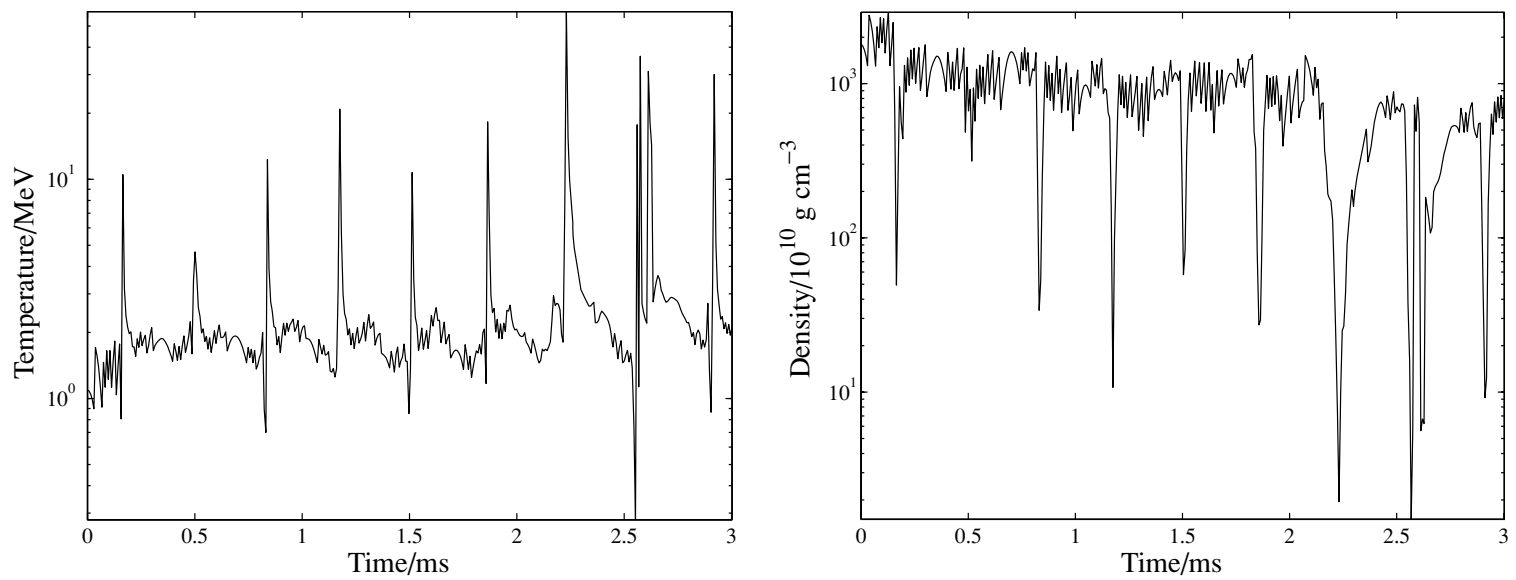

Figure 1. Left: neutrinosphere temperature vs. time. Right: neutrinosphere density vs. time. The initial mass of the neutron star is $1.55 M_{\odot}$.
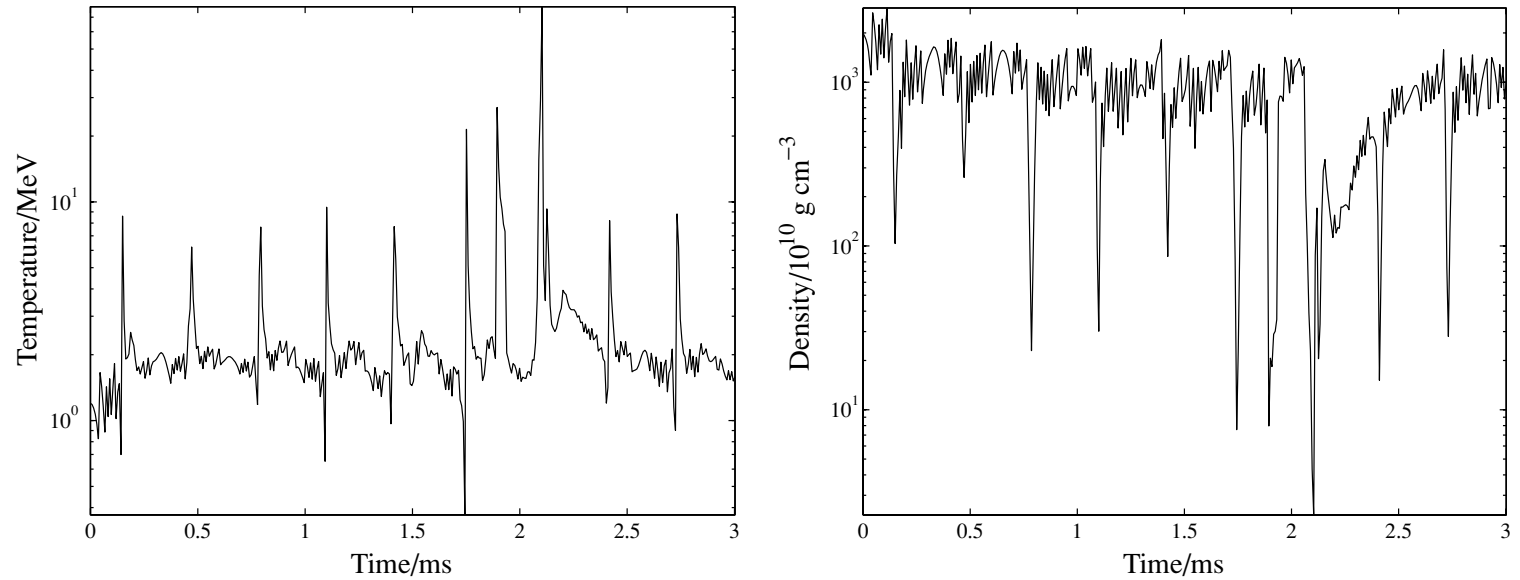

Figure 2. Left: neutrinosphere temperature vs. time. Right: neutrinosphere density vs. time. The initial mass of the neutron star is $1.7 M_{\odot}$.

there will always be a situation that the density is minimum and the temperature is maximum near the end of the first half of the cycle at the neutrinosphere.

Using $R_{v}$ and $T_{v}$ at the neutrinosphere obtained from the numerical simulation, we compute the time evolution of the neutrino luminosity. The results are shown in Figures 3 and 4 for two different neutron star masses, respectively. We can see that extremely intense neutrino pulses can occur when the temperature at the neutrinosphere is maximum while the density at the neutrinosphere is minimum. The pulsation period of the neutrino luminosity is equal to that of the temperature and density. In calculating the neutrino luminosity we have used the constraint $L_{v}<\dot{E}_{M}$, where $\dot{E}_{M}$ represents the rate of energy flow carried by a fluid into the cell containing the neutrinosphere $R_{\nu}$. Explicitly $\dot{E}_{M}=4 \pi R_{\nu}^{2} \Delta R \tau / \delta t$, where $\delta t$ is the dynamical timescale for the energy flow from one grid to another grid and $\tau=0.5 \rho v^{2}+\rho \epsilon$ is the total energy density at $R_{\nu}$.

The maximum neutrino luminosities range from $10^{53}$ to $10^{54} \mathrm{erg} \mathrm{s}^{-1}$. The total energies carried away by neutrinos in the period of first $3 \mathrm{~ms}$ are $6.75 \times 10^{49} \mathrm{erg}$ and $7.12 \times 10^{49} \mathrm{erg}$ for the star with $1.55 M_{\odot}$ and for the star with $1.75 M_{\odot}$, respectively, and the typical neutrino energy is $>30 \mathrm{MeV}$ during the peaks.

\subsection{Damping of the Oscillation}

With the grid resolution $(d x=0.28 \mathrm{~km})$ we used for the simulations, which is limited by the computational resource,

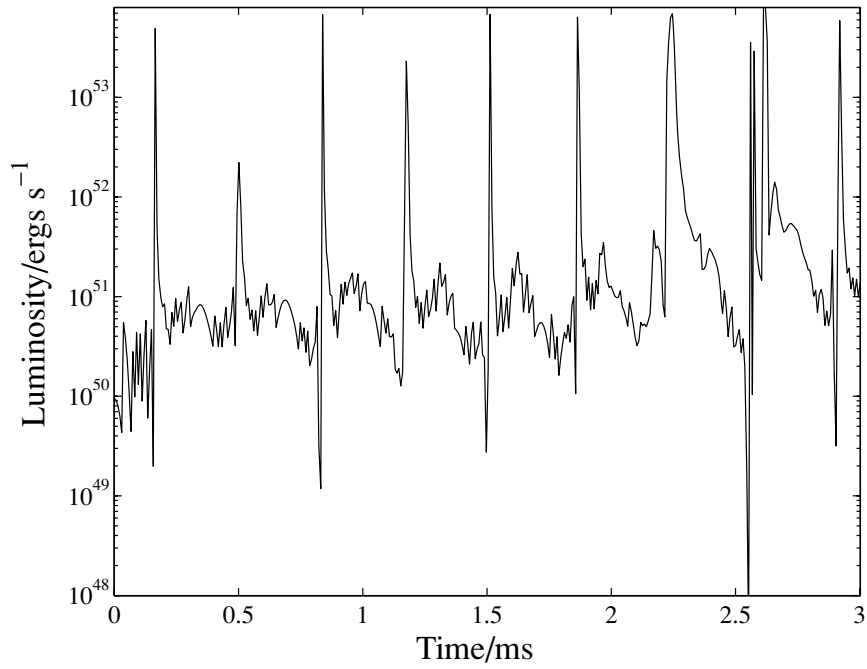

Figure 3. Neutrino luminosity as a function of time for a star with $1.55 M_{\odot}$.

we see that numerical damping becomes significant after about 3 ms. Recently, Abdikamalov et al. (2009) have used a twodimensional general relativistic numerical code to study the gravitational wave signals emitted from the phase-induced collapse of neutron stars and their results are very similar to our previous results (Lin et al. 2006). Since Abdikamalov et al. (2009) can achieve much higher resolution in two dimensions, they can perform longer timescale simulations with high 


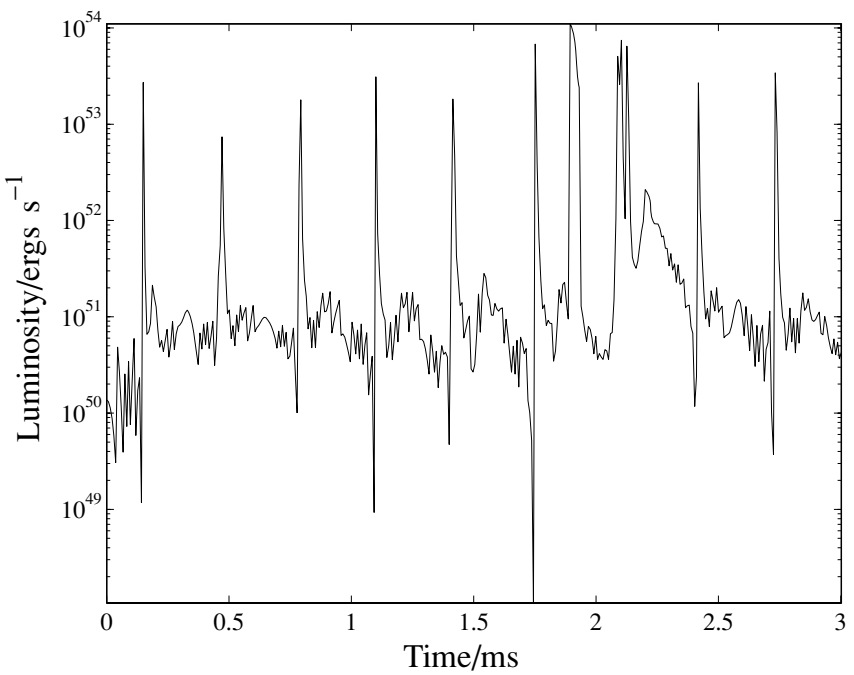

Figure 4. Neutrino luminosity as a function of time for a star with $1.7 M_{\odot}$.

accuracy. They conclude that the timescale of hydrodynamical damping effects (e.g., due to mass-shedding) is typically a few tens to hundreds of milliseconds.

However, there are still other physical damping effects which are not modeled in the simulations (Lin et al. 2006; Abdikamalov et al. 2009). Wang \& Lu (1984) first pointed out theoretically that the dissipation due to nonleptonic reaction is of great importance and the stellar pulsations of the quark stars would be strongly damped via the following process:

$$
s+u \leftrightarrow u+d
$$

According to Sawyer (1989) and Madsen (1992), in the hightemperature limit, which is exactly our case, the bulk viscosity can be obtained analytically:

$$
\begin{gathered}
\zeta=\frac{\alpha T^{2}}{\omega^{2}+\beta T^{4}}\left(1-\left(1-\exp \left(-\beta^{1 / 2} T^{2} \tau\right)\right) \frac{2 \beta^{1 / 2} T^{2} / \tau}{\omega^{2}+\beta T^{4}}\right) \\
\alpha=9.39 \times 10^{22} m_{s}^{4} \mu_{d}^{3} \mathrm{~g} \mathrm{~cm}^{-1} \mathrm{~s}^{-1} \\
\beta=7.11 \times 10^{-4} \mu_{d}^{6}\left(1+m_{s}^{2} / 4 \mu_{d}^{2}\right)^{2} \mathrm{~s}^{-2},
\end{gathered}
$$

where $m_{s}$ is the mass of strange quark mass in MeV, typically ranging from 100 to $300 \mathrm{MeV} . \mu_{d}$ is the down quark chemical potential; the typical value is around $235 \mathrm{MeV}$, assuming that the nuclear matter density is $2.8 \times 10^{14} \mathrm{~g} \mathrm{~cm}^{-3} \cdot \tau$ is the perturbation period and $\omega=2 \pi / \tau$.

For a star with relative constant density, Sawyer (1989) estimated the damping time of vibration:

$$
\tau_{D}=30^{-1} \rho R^{2} \zeta^{-1} .
$$

The average damping time is thus calculated with average temperature $(\langle T\rangle)$ and density $(\langle\rho\rangle)$, which are given by

$$
\begin{gathered}
\langle T\rangle=\frac{\int_{0}^{R_{\mathrm{tr}}} 4 \pi r^{2} T(r) \rho(r) d r}{\int_{0}^{R_{\mathrm{tr}}} 4 \pi r^{2} \rho(r) d r}, \\
\langle\rho\rangle=\frac{\int_{0}^{R_{\mathrm{tr}}} 4 \pi r^{2} \rho(r) d r}{\int_{0}^{R_{\mathrm{tr}}} 4 \pi r^{2} d r},
\end{gathered}
$$

where $R_{\mathrm{tr}}$ is the radius where the matter transits to quark matter, i.e., $\rho\left(R_{\mathrm{tr}}\right)=\rho_{\mathrm{tr}}$.

If we take $m_{s} \sim 140 \mathrm{MeV},\langle\rho\rangle \sim 10^{15} \mathrm{~g} \mathrm{~cm}^{-3}$, and $\langle T\rangle \sim$ $50 \mathrm{MeV}$, the damping timescale is $\sim 10 \mathrm{~s}$. This timescale is sufficiently long to allow the neutrinos to carry away most of the gravitational energy released from the phase-induced collapse neutron star $\left(\Delta E_{G} \sim G M^{2} \Delta R / R^{2}\right.$, where $\Delta R$ is the change of radius before and after the phase transition). For the model presented in this paper, $\Delta R / R$ is $\sim 0.2$, which gives $\sim 10^{53} \mathrm{erg}$, which does not conflict with the IMB results. Furthermore, in our simulation neutrinos can carry away $\sim 10^{50}$ erg in $3 \mathrm{~ms}$. If we assume that this is the only mechanism to damp out the oscillation energy, it only takes $3 \mathrm{~ms} \times \frac{10^{53} \mathrm{erg}}{10^{50} \mathrm{erg}} \sim 3 \mathrm{~s}$ to take this amount of energy away by neutrinos with energy $>30 \mathrm{MeV}$.

\section{COOLING OF QUARK STARS AND NEUTRON STARS}

The use of thermally excited helical vortex waves that produce fast magnetosonic waves in the stellar crust, and which propagate toward the surface and transform into outgoing electromagnetic radiation, has allowed the direct determination of the core temperature of neutron stars (Svidzinsky 2003). The core temperature of the Vela pulsar is $T=8 \times 10^{8} \mathrm{~K}$, while the core temperature of PSR B0656+14 and Geminga exceeds $2 \times 10^{8} \mathrm{~K}$. These temperature estimates rule out the EOSs incorporating Bose condensations of pions or kaons and superfluid quark matter in these objects. Thermal X-ray radiation from neutron star soft $\mathrm{X}$-ray transients in quiescence provides the strongest constraints on the cooling rates of neutron stars, and thus on the interior composition and properties of matter in the cores of compact objects (Heinke et al. 2007). The analysis of the new (2006) and archival (2001) $X M M-N e w t o n$ observations of the accreting millisecond pulsar SAX J1808.4-3658 in quiescence provides the most stringent constraints to date. Simultaneous fitting of all available $X M M$ data allows a constraint on the quiescent neutron star $(0.01-10 \mathrm{keV})$ luminosity of $L_{\mathrm{NS}}<1.1 \times 10^{31} \mathrm{erg} \mathrm{s}^{-1}$. This limit excludes some current models of neutrino emission mediated by pion condensates (Heinke et al. 2007), and provides further evidence for additional cooling processes, such as neutrino emission via direct Urca processes, involving nucleons in the cores of massive neutron stars. Hence, these recent observations show that the observed thermal luminosity of some neutron stars does not agree with some proposed exotic cooling processes (Tsuruta 1998; Yakovlev \& Haensel 2003; Yakovlev \& Pethick 2004).

\subsection{Energy Dissipation Mechanisms for Strange Stars}

Several physical processes that contribute to the energy emission from the bare quark star surface have been proposed. The most important of these energy loss mechanisms is electronpositron pair creation, due to the strong electric field at the surface of the strange star (Usov 1998a, 1998b). Other processes include neutral pion emission ( $\mathrm{Ng}$ et al. 2003), quark-quark bremsstrahlung (Chmaj et al. 1991; Cheng \& Harko 2003), equilibrium blackbody radiation (Chmaj et al. 1991), and electronelectron bremsstrahlung (Jaikumar et al. 2004; Harko \& Cheng 2005).

In the case of energy loss via the production of $e^{-} e^{+}$pairs, the energy flux is given by $F_{ \pm}=\varepsilon_{ \pm} \dot{n_{ \pm}}$, with $\varepsilon_{ \pm}=m_{e}+T$ and

$$
n_{ \pm}=\Delta r \frac{9 T^{3}}{2 \pi \varepsilon_{F}^{2}} \sqrt{\frac{\alpha}{\pi}} e^{-2 m_{e} / T} n_{e} J(\xi),
$$


where $\varepsilon_{F}=\left(\pi^{2} n_{e}\right)^{1 / 3}$ is the Fermi momentum of the electrons with number density $n_{e}, \alpha$ is the fine structure constant, $\xi=$ $2 \sqrt{\alpha / \pi}\left(\varepsilon_{F} / T\right), J(\xi)=(1 / 3) \xi^{3} \ln \left(1+2 \xi^{-1}\right) /(1+0.074 \xi)^{3}+$ $\left(\pi^{5} / 6\right) \xi^{4} /(13.9+\xi)^{4}$, and $\Delta r$ is the thickness of the electron layer (Usov 2001). Harko \& Cheng (2006) have considered the mean value of the $z$-dependent chemical potential, $\varepsilon_{F} \approx\left\langle\mu_{e}(z, T)\right\rangle \approx$ $(1 / d) \sqrt{3 / \alpha \pi}\left(V_{I} / T\right)\left(1+\sqrt{1+V_{I}^{2} / 2 \pi^{2} T^{2}}\right)^{-1}$. For temperatures of around $T=10 \mathrm{MeV}$ we obtain $\varepsilon_{F} \approx 3 \mathrm{MeV}$, a smaller value than that considered in Usov (2001), $\varepsilon_{F} \approx 18 \mathrm{MeV}$. This choice of the Fermi momentum significantly reduces the energy flux from electron-positron pair creation.

Pions are created at the stellar surface due to the collision between the quarks and the bag, representing the conversion of the quark kinetic energy to the mass of the pion cloud $(\mathrm{Ng}$ et al. 2003). The pions are assumed to decay via the processes $\pi^{0} \rightarrow 2 \gamma \longleftrightarrow e^{+}+e^{-}$and $\pi^{ \pm} \rightarrow \mu^{ \pm}+v_{\mu} \rightarrow e^{ \pm}+v_{e}+2 v_{\mu}$. The energy flux due to this process is given by $F_{\pi}=\rho_{\pi} v_{\pi}$, where $\rho_{\pi}$ is the energy density of the pion field at the star's surface, which is fixed by the axial current conservation, and $v_{\pi}$, the speed of the emitted pions, is $v_{\pi} \approx \sqrt{2 T / m_{\pi}}$, where $m_{\pi} \approx 140 \mathrm{MeV}$ is the mass of the pion ( $\mathrm{Ng}$ et al. 2003).

Electron-electron bremsstrahlung is an important energy loss mechanism for strange stars (Jaikumar et al. 2004; Harko $\&$ Cheng 2005). For surface temperatures $T<10^{9} \mathrm{~K}$, the photon flux exceeds that of the electron-positron pairs that are produced via the Schwinger mechanism in the presence of a strong electric field that binds electrons to the surface of the quark star. The average energy of photons emitted from the bremsstrahlung process can be $0.5 \mathrm{MeV}$ or more, which is larger than that in electron-positron pair annihilation. The effect of multiple and uncorrelated scattering on the radiation spectrum (the Landau-Pomeranchuk-Migdal effect), together with the effect of the strong electric field at the surface of the star, was discussed in Harko \& Cheng (2005). The presence of the electric field strongly influences the radiation spectrum emitted by the electrosphere. The radiation properties of the electrons in the electrosphere essentially depend on the value of the electric potential at the quark star surface. The effect of multiple scattering, which strongly suppresses radiation emission, is important only for the dense layer of the electrosphere situated near the star's surface, and only for high values of the surface electric potential of the star. The bremsstrahlung emissivity of the electrosphere can be obtained as

$$
\begin{aligned}
\varepsilon_{\mathrm{Br}}^{(\mathrm{ee})}(\mu, T)= & \frac{75}{4 \pi^{2}} \alpha r_{e}^{2} g^{2} \operatorname{Li}_{7 / 2}(\eta)\left[\left(\ln \frac{T}{2 m_{e}}+\frac{77}{30}-\gamma\right)\right. \\
& \left.\times \operatorname{Li}_{7 / 2}(\eta)+\left.\frac{d \operatorname{Li}_{n}(\eta)}{d n}\right|_{n=7 / 2}\right] T^{7},
\end{aligned}
$$

where $g=2$ is the statistical weight for electrons, $\eta=$ $-\exp \left[\mu_{e}(T) / T\right], \mathrm{Li}_{n}(\eta)=\sum_{k=1}^{\infty} \eta^{k} / k^{n}$ is the polylogarithm function, and $\gamma=0.577216$ is Euler's constant.

The bremsstrahlung energy flux from the exterior electron layer of the strange star, coming out from a thin surface layer of thickness $d z$, is $F_{\mathrm{Br}}^{(\mathrm{ee})}=\varepsilon_{\mathrm{Br}}^{(\mathrm{ee})} d z / \pi$. Taking into account the contribution of all layers we find

$$
F_{\mathrm{Br}}^{(\mathrm{ee})}(T)=\frac{1}{\pi} \int_{0}^{\infty} \varepsilon_{\mathrm{Br}}^{(\mathrm{ee})}(z, T) d z=\sigma_{\mathrm{Br}}^{(\mathrm{ee})}(T) T^{7}
$$

where

$$
\begin{aligned}
\sigma_{\mathrm{Br}}^{(\mathrm{ee})}(T)= & \frac{75}{4 \pi^{3}} \alpha r_{e}^{2} g^{2} \\
& \times \int_{0}^{\infty} \operatorname{Li}_{7 / 2}[\eta(z, T)]\left\{\left(\ln \frac{T}{2 m_{e}}+\frac{77}{30}-\gamma\right)\right. \\
& \left.\times \operatorname{Li}_{7 / 2}[\eta(z, T)]+\left.\frac{d \operatorname{Li}_{n}(\eta)}{d n}\right|_{n=7 / 2}\right\} d z .
\end{aligned}
$$

The energy flux from quark-quark bremsstrahlung can be represented as $F_{\mathrm{q}-\mathrm{q}}=\sigma_{\mathrm{Br}}\left(n_{b}, T\right) T^{4}$, where $\sigma_{\mathrm{Br}}\left(n_{b}, T\right) \approx$ $g^{2} L i_{2}^{2}\left[-\exp \left(\left(\pi^{2} n_{b}\right)^{1 / 3} / T\right)\right] I\left(n_{b}, T\right) \lambda /(2 \pi)^{3}, \lambda$ is the mean photon path in the quark matter, $n_{b}$ is the baryon number density at the surface of the star, and

$$
\begin{aligned}
\frac{\pi}{\alpha} I\left(n_{b}, T\right) \approx & \frac{1+3 \pi / 4 \alpha_{s}}{\tau} \ln \left[\frac{1+4\left(1+3 \pi / 4 \alpha_{s}\right)^{2}}{\left(1+4 \tau^{2} a^{2} n_{b}^{2 / 3}\right)^{a n_{b}^{1 / 3}}}\right] \\
& +\frac{1}{\tau}\left[\arctan 2\left(1+3 \pi / 4 \alpha_{s}\right)-\arctan 2 \tau a n_{b}^{1 / 3}\right] \\
& -4\left(\frac{1+3 \pi / 4 \alpha_{s}}{\tau}-a n_{b}^{1 / 3}\right) \\
& +\frac{1}{2 \tau}\left[D\left(1+3 \pi / 4 \alpha_{s}\right)-D\left(\tau a n_{b}^{1 / 3}\right)\right],
\end{aligned}
$$

where $\alpha_{s}$ is the strong coupling constant, $a=2^{1 / 3} 3^{4 / 3} \pi^{5 / 3} g^{2 / 3} e^{4}$ $Z^{2} \ln \left(184 Z^{-1 / 3}\right) / \alpha_{s}^{2}, \tau$ is the mean collision time between quarks given by

$$
\tau^{-1} \approx-n_{b}^{-1 / 3} T^{2} \operatorname{Li}_{2}\left[-\exp \left(\left(\pi^{2} n_{b}\right)^{1 / 3} / T\right)\right] / 8 \pi^{8 / 3},
$$

which can be roughly estimated as $\tau=1 / n \sigma_{0} v$, and the function $D(x)$ is defined as $D(x)=i\left[\mathrm{Li}_{2}(-2 i x)-\mathrm{Li}_{2}(2 i x)\right]$ (Cheng $\&$ Harko 2003). $\sigma_{0}$ is the quark-quark elastic scattering cross section. Due to interference between amplitudes of nearby interactions, the bremsstrahlung emissivity from the strange star surface is suppressed for frequencies smaller than a critical frequency (the Landau-Pomeranchuk-Migdal effect; Cheng \& Harko 2003). The range of the suppressed frequencies is a function of the quark matter density at the star's surface and of the QCD coupling constant. For temperatures much smaller than the Fermi energy of the quarks, the bremsstrahlung spectrum has the same temperature dependence as the equilibrium blackbody radiation. Multiple collisions could reduce the intensity of the bremsstrahlung radiation by an order of magnitude. The absorption in the semidegenerate electron gas can also significantly reduce the intensity of the quark-quark bremsstrahlung radiation and, consequently, the surface emissivity. The combined effects of multiple collisions and absorption in the electron layer could make the soft photon surface radiation of quark stars six orders of magnitude smaller than the equilibrium blackbody radiation (Cheng \& Harko 2003).

At temperature $T$ strange matter is filled with electromagnetic waves in thermodynamic equilibrium with quarks. The quanta of the electromagnetic waves in plasma (transverse plasmons) have a characteristic dispersion relation $\omega(k)=\sqrt{\omega_{p}^{2}+k^{2}}$, where $k$ is the wavenumber and the plasma frequency $\omega_{p}=$ $e\left(8 \pi n_{b} / 3 \mu\right)^{1 / 2}$ (Chmaj et al. 1991). The characteristic transverse plasmon cutoff frequency can be estimated as $\omega_{p} \approx 20$ $25 \mathrm{MeV}$ (Chmaj et al. 1991; Ng et al. 2003). A beam of transverse plasmons hitting the edge of strange star matter from inside will be partially reflected and will partially pass to the 


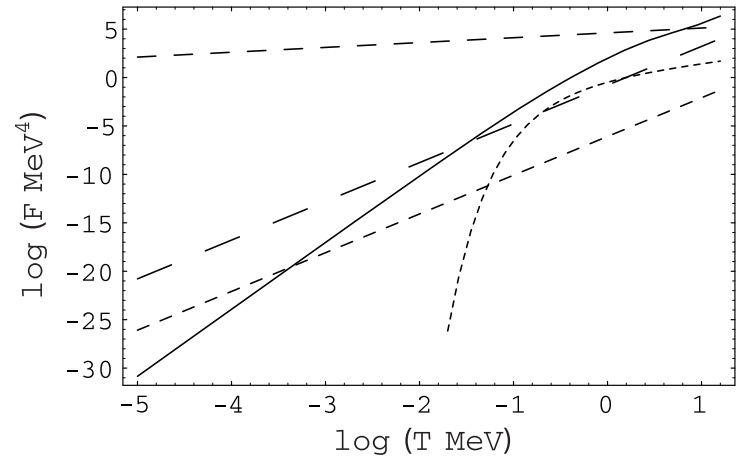

Figure 5. Temperature variation of the energy fluxes (in a logarithmic scale), emitted by the bare quark star surface, via different radiation mechanisms: electron-electron bremsstrahlung flux $F_{\mathrm{Br}}^{(\text {ee) }}$ (solid curve), electron-positron pair creation energy flux $F_{ \pm}$(dotted curve), quark-quark bremsstrahlung energy flux $F_{\mathrm{q}-\mathrm{q}}$ (dashed curve), energy flux $F_{\pi}$ due to pion emission (long dashed curve), and blackbody radiation energy flux $F_{\mathrm{bb}}$ (ultra-long dashed curve). For the surface electrostatic potential of the quark star we have chosen the typical value $V_{I}=14 \mathrm{MeV}$, while the thickness of the electron layer was taken as $d=1$ $000 \mathrm{fm}$. For the electron-positron energy flux, the Fermi energy of the electrons $\varepsilon_{F}=18 \mathrm{MeV}=$ constant. For the energy density of the pion field at the strange star surface we have adopted the value $\rho_{\pi} \approx 3.417 \times 10^{5} \mathrm{MeV}^{4} \approx 7.1 \times 10^{31}$ erg $\mathrm{cm}^{-3}$.

outer vacuum, being refracted. The energy flux of thermal equilibrium photons radiated from the bare strange surface is given by

$$
F_{\mathrm{eq}}=\frac{1}{2} \int_{\omega_{p}}^{\infty} \omega\left(\omega^{2}-\omega_{p}^{2}\right) g(\omega)\left[e^{\omega / T}-1\right]^{-1} d \omega,
$$

where $g(\omega)=\left(1 / 2 \pi^{2}\right) \int_{0}^{\pi / 2}\left[1-\left(R_{\perp}+R_{\|}\right) / 2\right] \sin \theta \cos \theta d \theta$, with $R_{\perp}=\sin ^{2}\left(\theta-\theta_{0}\right) / \sin ^{2}\left(\theta+\theta_{0}\right)$ and $R_{\|}=\tan ^{2}\left(\theta-\theta_{0}\right) /$ $\tan ^{2}\left(\theta+\theta_{0}\right) . \theta_{0}$ is defined as $\theta_{0}=\arcsin \left[\sin \theta \sqrt{1-\left(\omega_{p} / \omega\right)^{2}}\right]$, respectively (Chmaj et al. 1991).

The variations of the energy fluxes $F$ for these energy mechanisms are represented as a function of temperature in Figure 5.

When the temperature of the quark star core drops below $10^{9}$ $\mathrm{K}$, the strange matter becomes superfluid. At this temperature, quarks can form colored Cooper pairs near the Fermi surface and become superconducting. From the BCS theory it follows that the critical temperature $T_{c}$ at which the transition to the superconducting state takes place is $T_{c}=\Delta / 1.76$, where $\Delta$ is the pairing gap energy (Blaschke et al. 2000). An early estimation of $\Delta$ gave $\Delta \sim 0.1-1 \mathrm{MeV}$ (Bailin \& Love 1984), but some recent studies considering instanton-induced interactions between quarks estimated $\Delta \sim 100 \mathrm{MeV}$ (Alford et al. 1999; Rajagopal \& Wilczek 2001). When the temperature of the star is below $T_{c}$, the emissivity of the quark matter is modified by the superconducting effects. Since the collisions between the quarks and the bag are suppressed, pion emissivity is suppressed by a factor of $\exp (-\Delta / T)(\mathrm{Ng}$ et al. 2003). The same factor can be used to describe the suppression of quark-quark bremsstrahlung due to the superconductivity of the strange matter.

Figure 6 shows the variation with temperature of the energy fluxes for various dissipation mechanisms if quarks become superfluid.

For $T \ll \omega_{p}$ the equilibrium photon emissivity of strange matter is negligibly small compared to the blackbody spectrum. Even for a small value of the gap pairing energy $\Delta$, the superfluidity of the quark matter strongly suppresses the quarkquark bremsstrahlung and pion emissivity of the star.

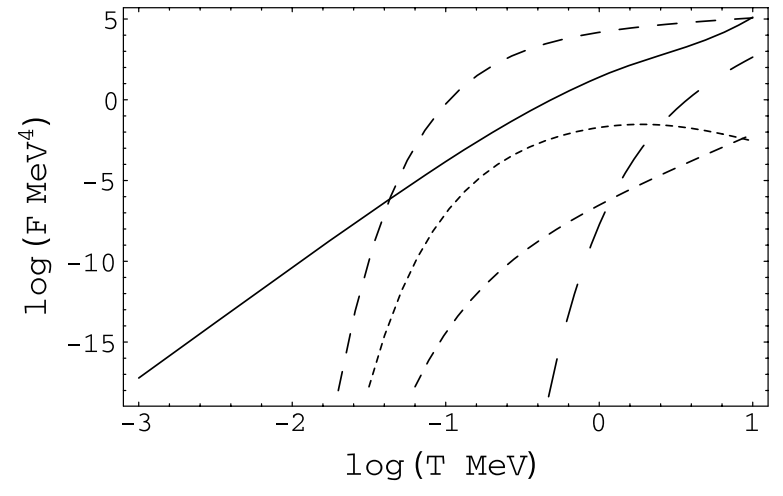

Figure 6. Temperature variation of the energy fluxes (in a logarithmic scale), emitted by the superfluid bare quark star surface, via different radiation mechanisms: electron-electron bremsstrahlung flux $F_{\mathrm{Br}}^{(\mathrm{ee})}$ (solid curve), electron-positron pair creation energy flux $\left\langle F_{ \pm}\right\rangle$(dotted curve), quark-quark bremsstrahlung energy flux $F_{\mathrm{q}-q}^{\text {(sup) }}$ (dashed curve), energy flux $F_{\pi}^{\text {(sup) }}$ due to pion emission (long dashed curve), and the thermal photon equilibrium radiation energy flux $F_{\text {eq }}$ (ultra-long dashed curve). For the surface electrostatic potential of the quark star we have chosen the typical value $V$, while the thickness of the electron layer was taken as $d=1000 \mathrm{fm}$. The energy gap $\Delta=1 \mathrm{MeV}$.

Strange quark matter in the CFL phase of QCD, which occurs for large gaps $(\Delta \sim 100 \mathrm{MeV})$, could be rigorously electrically neutral, despite the unequal quark masses, even in the presence of the electron chemical potential (Alford et al. 1999; Rajagopal $\&$ Wilczek 2001).

However, Page \& Usov (2002) pointed out that for sufficiently large $m_{s}$ the low-density regime is rather expected to be in the "two-color-flavor superconductor" phase in which only $u$ and $d$ quarks of two colors are paired in a single condensate while those of the third color and $s$ quarks of all three colors are unpaired. In this phase, electrons are present. In other words, electrons may be absent in the core of strange stars but present, at least, near the surface where the density is lowest. Nevertheless, the presence of CFL effect can reduce the electron density at the surface and hence also significantly reduces the bremsstrahlung emissivity of the electrons in the surface layer.

\subsection{Cooling of Strange Stars}

In this subsection, we consider the cooling of a quark star. For simplicity, we assume that the star is of uniform density and isothermal, that is, the core and surface temperatures are equal. The effect of the magnetic field on the cooling is also neglected. The thermal evolution of the quark star is determined by the equation

$$
C_{V} \frac{d T}{d t}=-\sum_{i=1}^{n} L_{i}=-\left(L_{\mathrm{pair}}+L_{\mathrm{qq}}+L_{\mathrm{ee}}+L_{\pi}+L_{\mathrm{eq}}+L_{v}\right)
$$

where $C_{V}$ is the specific heat, $T$ is the temperature, and $L_{\text {pair }}$ is the electron-positron pair luminosity, $L_{\mathrm{qq}}$ is the quarkquark bremsstrahlung luminosity, $L_{\mathrm{ee}}$ is the electron-electron bremsstrahlung luminosity, $L_{\pi}$ is the pion emission luminosity, $L_{\mathrm{eq}}$ is the luminosity due to the thermal equilibrium radiation, and $L_{v}$ is the neutrino luminosity, respectively. In the normal state of quark matter, the quark Fermi momentum $p_{\mathrm{Fq}}$ can be approximated as $p_{\mathrm{Fq}}=235\left(\rho / \rho_{0}\right)^{1 / 3} \mathrm{MeV} / c$, where $\rho_{0}$ is the nuclear density, and the specific heat of the quark matter is given by $c_{q}=2.5 \times 10^{20}\left(\rho / \rho_{0}\right)^{2 / 3} T_{9} \mathrm{erg} \mathrm{cm}^{-3} \mathrm{~K}^{-1}$ (Iwamoto 1980). In the superfluid state, for $0.2 T_{c} \leqslant T \leqslant T_{c}$, the specific heat can be obtained as $c_{q}^{\text {(sf) }}=3.15 c_{q}\left(T_{c} / T\right) \exp \left(-1.76 T_{c} / T\right)[2.5-$ 
$\left.1.66\left(T / T_{c}\right)+3.64\left(T / T_{c}\right)^{2}\right]$, while for $T<0.2 T_{c}$ the specific heat is zero (see $\mathrm{Ng}$ et al. 2003, and references therein). The Goldstone excitations in the quark-gluon plasma contribute to the specific heat of the strange star, so that $c_{g-\gamma}=3.0 \times$ $10^{13} N_{g-\gamma} T_{9}^{3}$ erg $\mathrm{cm}^{-3} \mathrm{~K}^{-1}$, where $N_{g-\gamma}$ is the number of available massless gluon-photon states, which are present even in the color superconducting phase (Blaschke et al. 2000). When the temperature is low, the specific heat of quark matter vanishes. However, the electrons are not affected, and at low temperatures their effect is important. The specific heat of the electrons is given by $c_{e}=1.7 \times 10^{20}\left(Y_{e} \rho / \rho_{0}\right)^{2 / 3} T_{9} \mathrm{erg} \mathrm{cm}^{-3} \mathrm{~K}^{-1}(\mathrm{Ng}$ et al. 2003), where $Y_{e} \approx 10^{-3}$ is the electron fraction.

Neutrinos are emitted by quark matter through the URCA process, $d \rightarrow u+e^{-}+\bar{v}_{e^{-}}, u+e^{-} \rightarrow d+v_{e^{-}}$. The neutrino emissivity for this process can be obtained as $\varepsilon_{d} \simeq 8.8 \times 10^{26} \alpha_{c}\left(\rho / \rho_{0}\right) Y_{e}^{1 / 3} T_{9}^{6}$ erg $\mathrm{cm}^{-3} \mathrm{~s}^{-1}$ (Iwamoto 1980). In the superfluid state, the neutrino emissivity is suppressed by a factor of $\exp (-\Delta / T)$. The exact form for the neutrino emissivity can be obtained as $\varepsilon_{d}=$ $\left(457 \pi / 840 \hbar^{10} c^{9}\right) G_{F}^{2} \cos ^{2} \theta_{C}\left(1-\cos \theta_{u e} / a\right) P_{F u} P_{F d} P_{F e}\left(k_{B} T\right)^{6}$ erg cm $\mathrm{cm}^{-3} \mathrm{~s}^{-1}$ (Duncan et al. 1983), where $G_{F}$ is the Fermi weak coupling constant, $\theta_{C}$ is the Cabibbo angle, $a=$ $\left(1-2 \alpha_{c} / \pi\right)^{-1 / 3}, P_{F u}, P_{F d}$, and $P_{F e}$ are the Fermi momenta of the $u, d$ quarks, and of the electrons, respectively. The angle between the $u$ and $e$ momenta, $\theta_{u e}$, is given by $\cos \theta_{u e}=$ $\left(P_{F d}^{2}-P_{F u}^{2}-P_{F e}^{2}\right) / 2 P_{F u} P_{F e}$. The condition for the URCA process to occur is $\left|\cos \theta_{u e}\right| \leqslant 1$ ( $\mathrm{Ng}$ et al. 2003). In the case of the similar reactions for the $s$ quarks, $s \rightarrow u+e^{-}+\bar{v}_{e^{-}}$, $u+e^{-} \rightarrow s+v_{e^{-}}$, the condition $\left|\cos \theta_{u e}^{\prime}\right| \leqslant 1$, where the angle $\theta_{u e}^{\prime}$ is defined as $\cos \theta_{u e}^{\prime}=\left(P_{F s}^{2}-P_{F u}^{2}-P_{F e}^{2}\right) / 2 P_{F u} P_{F e}$, is satisfied only if the density of the quark matter is as high as $4 \times 10^{16} \mathrm{~g} \mathrm{~cm}^{-3}$. Therefore, these reactions do not exist in the quark stars. The neutrino emissivity due to the photon-gluon mixing, which can generate a massive photon-gluon excitation, is neglected, since it is important only at temperatures higher than $70 \mathrm{MeV}$ (Blaschke et al. 2000). The cooling curve and the luminosity curve of the $1.55 M_{\odot}$ strange star are presented as the solid curve in Figures 7 and 8, respectively. We can see the radiation luminosity at $t \sim 20$ years is below $10^{34} \mathrm{erg} \mathrm{s}^{-1}$ but with a high temperature $T \sim 10^{7} \mathrm{~K}$ and the spectrum is a thermal bremsstrahlung.

\subsection{Neutron Star Cooling Process}

In this subsection, we describe a simple cooling model of neutron stars including a possible exotic matter core, e.g., meson condensate or quark core (see, e.g., Cheng et al. 1992, Chong $\&$ Cheng 1993). We assume that the neutron star with $1.55 M_{\odot}$ and $10^{6} \mathrm{~cm}$ in radius has uniform density, and has an isothermal core temperature $\left(T_{c}\right)$. We adopt the simple relation between the core temperature and the surface temperature $\left(T_{s}\right)$ given by the relation $T_{c 8}=1.3\left(T_{s 6}^{4} / g_{s 14}\right)^{0.445}$ (Gudmundsson et al. 1983). The core temperature evolution of the star is given by

$$
C_{v} d T_{c} / d t=-L_{v}-L_{\mathrm{bb}}\left(T_{s}\right)
$$

The heat capacity $C_{v}$ is given by (Maxwell 1979)

$$
C_{v}=C^{(e)}+C^{(n)}
$$

where

$$
C^{(e)}=1.9 \times 10^{37} M_{\odot} \rho_{14}^{1 / 3} T_{9} \mathrm{erg} \mathrm{K}^{-1}
$$

is the electron heat capacity with $\rho_{14}=\rho / 10^{14} \mathrm{~g} \mathrm{~cm}^{-3}$ and $T_{9}=T / 10^{9} \mathrm{~K}$, and

$$
\begin{aligned}
& C^{(n)} \\
& \quad \begin{array}{l}
C_{n}(T)=2.3 \times 10^{39} M_{\odot}\left(\frac{m_{n}^{*}}{m_{n}}\right) \rho_{14}^{-2 / 3} T_{9} \mathrm{erg} \mathrm{K}^{-1} \\
\quad \text { for } T>T_{n} \\
3.15 C_{n}(T)\left(\frac{T_{n}}{T}\right) e^{-1.76 T_{n} / T}\left[2.5-1.66 \frac{T}{T_{n}}+3.64\left(\frac{T}{T_{n}}\right)^{2}\right] \\
\quad \operatorname{erg~K} \mathrm{K}^{-1} \text { for } T \leqslant T_{n}
\end{array}
\end{aligned}
$$

is the neutron heat capacity, with $T_{n}$ being the transition temperature of normal-superfluid neutrons and $m_{n}^{*}$ the effective mass of neutrons. We adopted $m_{n}^{*} / m_{n} \simeq 1$ and $T_{n}=3.2 \times$ $10^{9} \mathrm{~K}$ (Takatsuka \& Tamagaki 1971).

The major neutrino emissions are due to the modified URCA process and $N N$-bremsstrahlung, where $N$ is a nucleon, $n$ or $p$. These mechanisms are relatively weak and the luminosity can be approximated by (Yakovlev \& Haensel 2003)

$$
L_{v}=L_{s} T_{9}^{8},
$$

where $L_{s}$ is at the range of $4 \times 10^{38}$ to $10^{40} \mathrm{erg} \mathrm{s}^{-1}$.

When considering some exotic neutrino processes, the emission is greatly enhanced. The neutrino luminosity can be written as (Yakovlev \& Haensel 2003)

$$
L_{v}=L_{f} T_{9}^{6},
$$

where $L_{f}$ lies at the range $4 \times 10^{41}$ to $4 \times 10^{44} \mathrm{erg} \mathrm{s}^{-1}$ for the pion condensation process and $4 \times 10^{41}$ to $4 \times 10^{43} \mathrm{erg} \mathrm{s}^{-1}$ for the kaon condensation and quark cooling processes.

The cooling curves and the radiation luminosities of neutron star with various cooling mechanisms are shown in Figures 7 and 8 , respectively. We can see that for neutron stars without exotic cooling processes, e.g., pion condensation or kaon condensation, their thermal luminosity is much larger than $10^{34} \mathrm{erg} \mathrm{s}^{-1}$. But the exotic cooling processes can cool the star much faster and the thermal luminosity can be much lower than $10^{34} \mathrm{erg} \mathrm{s}^{-1}$. On the other hand, the surface temperature of the neutron star with exotic cooling processes is very low, $<10^{6} \mathrm{~K}$, and it is a thermal spectrum.

\section{DISCUSSIONS AND FINAL REMARKS}

The detection of neutrinos from SN 1987A has suggested that a compact object should be formed at the center of SN 1987A. If the time delay and energy distribution difference between neutrinos detected by KAM-II and IMB are genuine together with the absence of hot compact object at the center of 1987A, which implies a rapidly cooling cold compact object, we have proposed that the neutrinos detected by IMB may result from a delay phase-induced collapse after the neutron star is formed. The final compact object may be a neutron star with exotic matter core or a strange star; either of them is a rapidly cooling object. Consequently, the current radiation luminosity from SN 1987A should be much lower than $10^{34} \mathrm{erg} \mathrm{s}^{-1}$. However, we have argued that the phase transition from neutron star to strange star may be the most possible phase transition occurring in 1987A. Such a collapse process can generate temperature and density oscillation on the neutrinosphere. By using a three-dimensional Newtonian code with a toy model, in which the phase transition is taken to be instantaneous and the EOS is assumed to be a mixed phase, we find that intense pulsating neutrinos with 


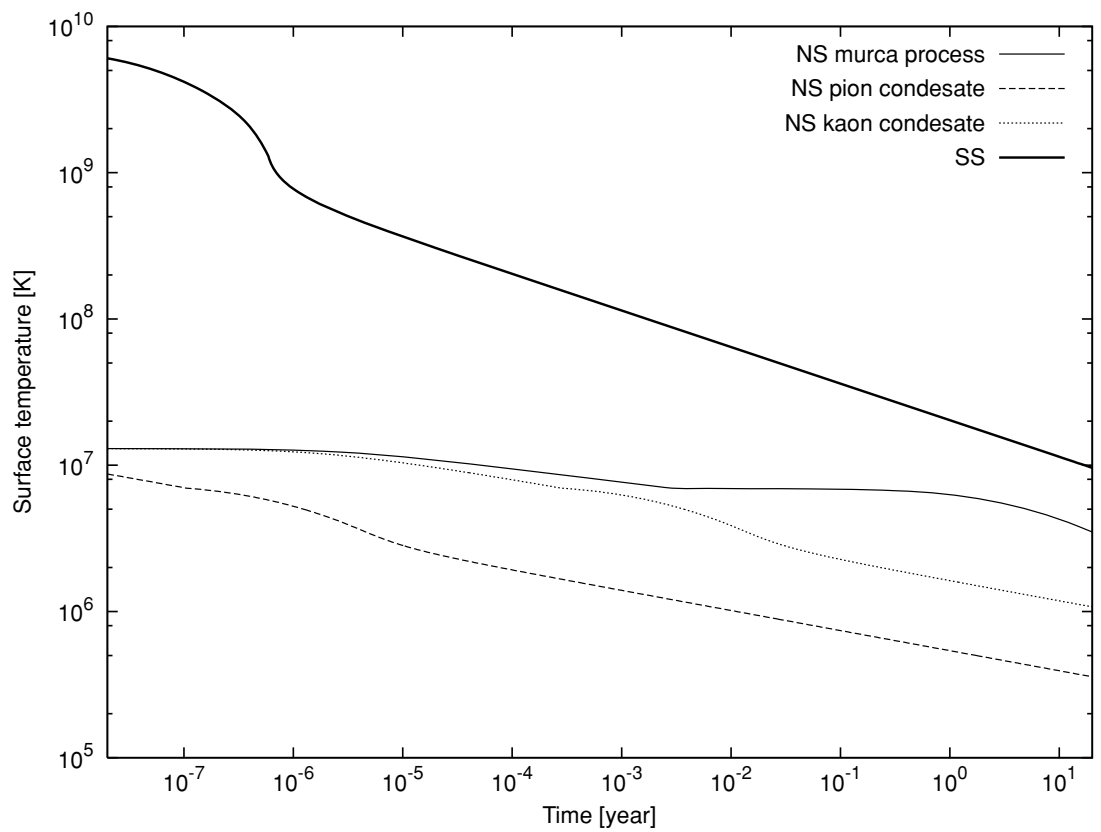

Figure 7. Surface temperature as a function of time. The heavy solid line is the bare strange star cooling curve. The light solid line, the dotted line, and the dashed line are the cooling curves of the neutron star with modified URCA, kaon condensate, and pion condensate, respectively.

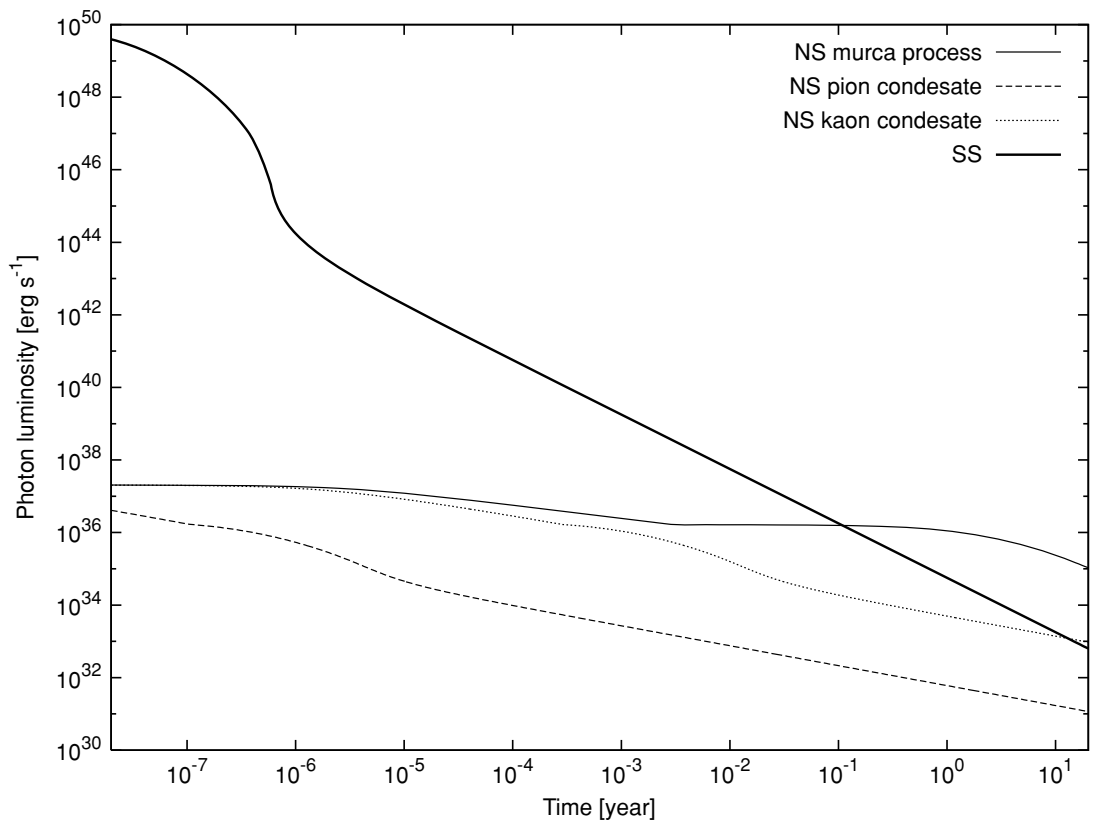

Figure 8. Surface photon luminosity as a function of time. The heavy solid line is the bare strange star cooling curve. The light solid line, the dotted line, and the dashed line are the cooling curves of the neutron star with modified URCA, kaon condensate, and pion condensate, respectively.

energy $>30 \mathrm{MeV}$ can be emitted. This phenomenon should be generic because the compression generates more heat in the core and the heat flow is outgoing during the contraction phase, whereas the matter is flowing in. That should create a situation where the temperature is maximum and the density is minimum at the neutrinosphere. It is very interesting to detect intense pulsating hot neutrino emission from supernova explosions occurring from nearby galaxies. Future neutrino experiments, such as ANTARES and IceCube (see Halzen 2006 for a review), may be able to detect such signals.

In calculating the cooling of a strange star, we have assumed a bare strange star, i.e., a strange star without crust. This assumption may be justified for the following reasons: on the top of the quark matter, a thin crust can exist as long as the electron density within it is smaller than that in the quark matter (Alcock et al. 1986). Such a baryonic crust is, however, much thinner than the neutron star crust. The mass of the crust on the quark star is at most $10^{-5} M_{\odot}$, because the density of the crust bottom cannot exceed neutron drip density, and it may extend to up to $250-300 \mathrm{~m}$. The normal crust may occur, for instance, due to the accretion of normal matter onto a bare strange star. The oscillation spectrum of strange stars with crust differs from the spectrum of neutron stars (Chuganov 2006). If detected, acoustic oscillations would allow one to discriminate between strange stars with crust and neutron stars, and constrain the mass and radius of the star. The recent detection of seismic 
vibrations in the aftermath of giant flares from two magnetars (highly magnetized compact stars) is a major breakthrough in observational astronomy. The oscillations excited seem likely to involve the stellar crust, the properties of which differ dramatically for strange stars. The resulting mode frequencies for strange stars cannot be reconciled with the observations, for reasonable magnetar parameters (Watts \& Reddy 2007). On the other hand, young and hot strange stars are the source of a powerful pair wind, consisting of electron-positron pairs and photons, created by the Coulomb barrier at the quark surface (Aksenov et al. 2005). Photons dominate in the emerging emission, and the emerging photon spectrum is rather hard and differs substantially from the thermal spectrum expected from a neutron star with the same luminosity. The total luminosity in the case of a stationary, spherically outflowing, pair wind is in the range of $L=10^{35}-10^{42} \mathrm{erg} \mathrm{s}^{-1}$. These results have direct relevance to the emission from hot, bare, strange stars. For $L>2 \times 10^{35} \mathrm{erg} \mathrm{s}^{-1}$, photons dominate the emerging emission. As $L$ increases from $10^{35}$ to $10^{42} \mathrm{erg} \mathrm{s}^{-1}$, the mean photon energy decreases from $\sim 400-500 \mathrm{keV}$ to $40 \mathrm{keV}$, while the spectrum changes in shape from a wide annihilation line to being nearly blackbody with a high-energy (greater than $100 \mathrm{keV}$ ) tail. Such a correlation of the photon spectrum with the luminosity, together with the fact that super-Eddington luminosities can be achieved, might be a good observational signature of hot, bare, strange stars (Aksenov et al. 2003). At the moment of its formation, a strange star is very hot. The temperature of the interior may be as high as a few $10^{11} \mathrm{~K}$. The neutrino luminosity of the young quark star is of the order of $10^{54} \mathrm{erg} \mathrm{s}^{-1}$, the rate of mass ejection from such a hot compact object is very high, and the normal matter envelope is blown away by radiation pressure in a few seconds. High temperatures also lead to a considerable reduction of the Coulomb barrier, increasing the tunneling of nuclei through the barrier toward the surface. Therefore, it is natural to expect that the surface of a young strange star will be nearly (or completely) bare (Usov 2001). Since the strange quark matter at the surface of the star is bound via strong interactions rather than gravity, such a star can radiate at the luminosity greatly exceeding the Eddington limit. Therefore, due to the radiation pressure, a normal matter crust cannot be built around a young strange star.

We have also assumed that the phase transition should take place after the neutron star is formed. We argue that the delay timescale of the phase transition is of the order of the neutrino trapping timescale for the following reasons: the theory of type II supernovae predicts the gravitational collapse of a degenerate core on timescales of the order of $t_{\text {coll }} \sim(G \rho)^{-1 / 2} \sim$ $4 \times 10^{-3} \rho_{12} s \sim 0.1 \mathrm{~s}$, followed by the formation of a hot $\left(k_{B} T \sim 50 \mathrm{MeV}\right)$ proto-neutron star, with a radius of around $50 \mathrm{~km}$, composed of $N_{b} \sim 10^{57}$ baryons, and with electron lepton number $L_{e} \approx 0.35 N_{b}$ (Haensel et al. 2007). The initial mass of the collapsing core is $M_{\text {core }} \approx N_{b} m_{0}$, where $m_{0}=$ $1.6586 \times 10^{-24} \mathrm{~g}$ is the mass of the ${ }^{56} \mathrm{Fe}$ nucleus, divided by 56 . The gravitational mass of the proto-neutron star is only slightly lower than $M_{\text {core }}$, because the gain in the gravitational energy is compensated by the increase of the internal energy contained mostly in the strongly degenerate neutrinos $v_{e}$, trapped in the stellar interior. The diffusion timescale of the neutrinos may be estimated by assuming that coherent scattering is the dominant opacity source, so that $t_{\text {diff }} \approx \lambda_{A}^{\text {coh }} N_{\text {scatt }} / c$, where $\lambda_{A}^{\text {coh }}$ is the mean free path in the sea of heavy nuclei $(A, Z)$ and $N_{\text {scatt }}>1$ is the number of scatterings experienced by the neutrino prior to escape. By taking into account the explicit expressions of the neutrino mean free path and of the number of scatterings, the neutrino diffusion time can be written as $t_{\text {diff }} \sim 0.08 \rho_{12} \mathrm{~s}$. At sufficiently high densities $t_{\text {diff }} \gg t_{\text {coll }}$ (Shapiro \& Teukolsky 1983). The two timescales becomes comparable at $t_{\text {diff }} \sim t_{\text {coll }}$, a condition which is satisfied at a density of $\rho_{\text {trap }} \sim 1.4 \times$ $10^{11} \mathrm{~g} \mathrm{~cm}^{-3}$.

Neutrino trapping has enormous implications for the core collapse. For $\rho \geqslant \rho_{\text {trap }}$, most of the neutrinos from electron capture remain in the matter, and the lepton number per baryon does not change. The neutrino distribution approaches an equilibrium Fermi-Dirac distribution. The neutrino luminosities are greatly reduced by trapping. Once the center of the core exceeds nuclear densities of the order of $\rho_{\text {nucl }}=2.8 \times 10^{14}$ $\mathrm{g} \mathrm{cm}^{-3}$, thermal pressure and nuclear forces cause the EOS of the matter to stiffen, preventing further collapse. Most of the gravitational binding energy of the core is released in the form of neutrinos, following the collapse to nuclear densities. In the absence of neutrino trapping, the total binding energy would be completely emitted as neutrinos in a collapse timescale, the time for the core to contract from $2 R_{\text {nucl }}$ to $R_{\text {nucl }}$, where $R_{\text {nucl }} \sim 12 \mathrm{~km}$ for $M \sim M_{\odot}$. Accordingly, the neutrino luminosity would then achieve its maximum possible value, $L_{v, \text { max }}=G M^{2} / R_{\text {nucl }} / t_{\text {coll }} \sim 10^{57} \mathrm{erg} \mathrm{s}^{-1}$ (Shapiro $\&$ Teukolsky 1983). In reality, neutrino trapping forces the liberated gravitational potential energy to be emitted on a much longer diffusion timescale, $t_{\text {diff }} \gg t_{\text {coll }}$ at $\rho \sim \rho_{\text {nucl }}$. As a result, the actual neutrino luminosity is of the order of $L_{v}=$ $G M^{2} / R_{\text {nucl }} / t_{\text {diff }} \sim 10^{52} \mathrm{erg} \mathrm{s}^{-1}$. Thus during the advanced stages of the collapse, the neutrinos are unable to stream freely out of the core. The bulk of the liberated gravitational energy must therefore be converted into other forms of internal energy (e.g., thermal energy, energy of the excited nuclear states, bounce kinetic energy), rather than being released immediately in the form of escaping neutrinos.

In order for the phase transition to take place in the neutron star, the density inside the core must satisfy the condition $\rho \geqslant \rho_{\text {trans }}$, as discussed in Section 2. Since the core temperature during the neutrino trapping period is very high, the thermal pressure will give a significant contribution to the total pressure, thus lowering the central density of the neutron star, so that $\rho<\rho_{\text {trans. }}$. In order to satisfy the thermodynamical conditions for the phase transition, the core must cool down so that the core density increases. To estimate the density increase resulting from the temperature decrease in the core of the neutron star, we solve the TOV equation by using a temperature-dependent EOS, i.e., Shen et al. (1998), to obtain core density as a function of temperature for a fixed stellar mass. In implementing this EOS, we have added an ideal electron gas contribution. We have also imposed the condition of beta equilibrium to determine the electron fraction. The left panel of Figure 9 shows the energy density profiles for a star model with $M=1.55 M_{\odot}$ at different temperatures. Note that the temperature profiles are determined by the general relativistic isothermal condition. The right panel of Figure 9 shows the results for a neutron star with $M=1.7 M_{\odot}$. In Figure 9, we can see that indeed when core temperature decreases by a factor of 2 , the core energy density can increase by more than $20 \%$. We can see that these changes are sufficiently large to trigger the phase transition when the core temperature decreases after the neutrino trapping timescale.

We conclude that the abnormally high energy neutrinos and the time delay detected by the IMB detector can be explained by neutrino trapping in the newly formed very hot neutron star, followed by a phase transition. After phase transition if the 

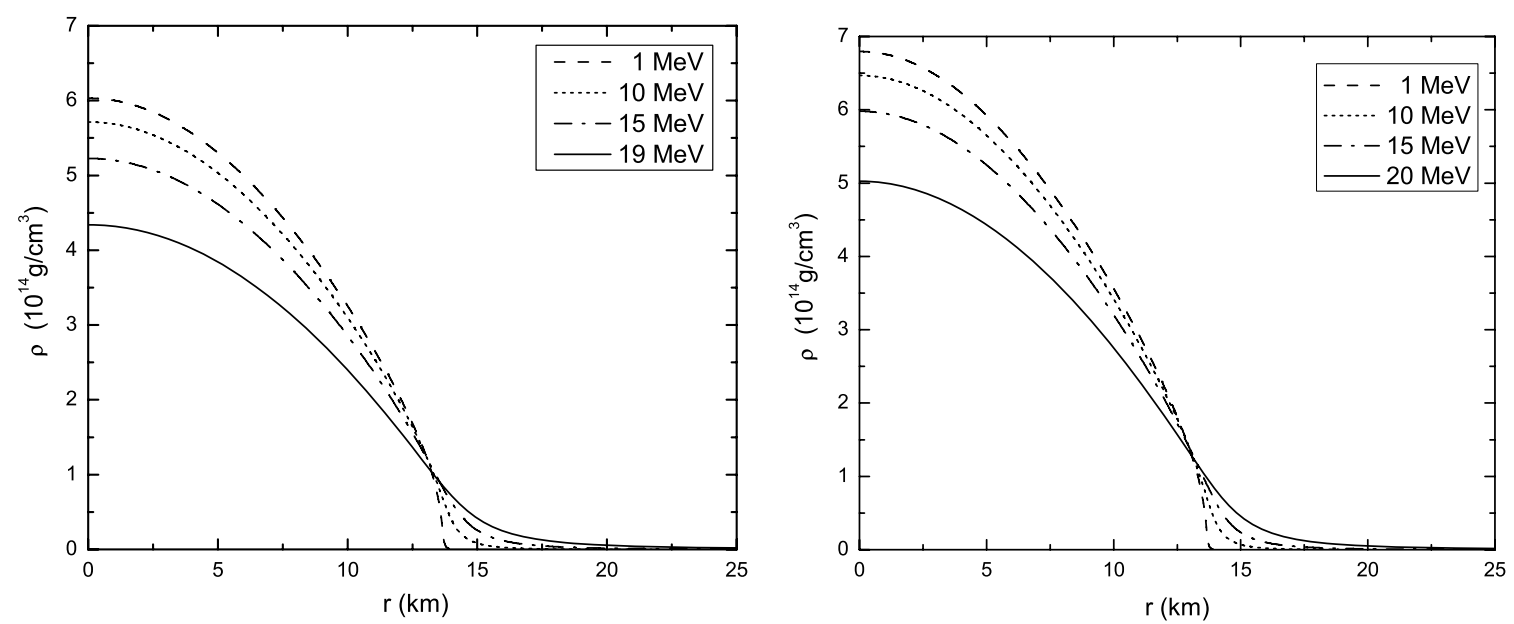

Figure 9. Left: the energy density profile of stars with mass $1.55 M_{\odot}$ at different surface temperatures $T_{s}=1,10,15,19 \mathrm{MeV}$. The central temperatures of the stars are $T_{c}=1.49,14.7,21.5,26.0 \mathrm{MeV}$, respectively. Right: the energy density profile of stars with mass $1.7 M_{\odot}$ at different surface temperatures $T_{s}=1,10,15$, $20 \mathrm{MeV}$. The central temperatures of the stars are $T_{c}=1.57,15.5,22.8,28.9 \mathrm{MeV}$, respectively.

compact object is a neutron star with exotic matter core, its surface luminosity is lower than $10^{34} \mathrm{erg} \mathrm{s}^{-1}$ and it should have blackbody thermal spectrum with temperature $<10^{6} \mathrm{~K}$. On the other hand if it is a bare strange star, its surface luminosity is also lower than $10^{34} \mathrm{erg} \mathrm{s}^{-1}$ but the radiation spectrum is a thermal bremsstrahlung spectrum with temperature $>10^{7} \mathrm{~K}$. However, the nature of the phase transition cannot be inferred from the present data, since the observed features cannot differentiate or discriminate between the different phase-transition models. We suggest that future observations on spectral features of the compact remnant of 1987A could provide better clues to elucidate the nature of the phase transition that might take place in SN1987A.

We thank Professors Z. G. Dai, P. Haensel, Y. F. Huang, Kwong Lau, K.B. Luk, C. S. Pun, and V. Usov for useful comments, and the anonymous referee for very useful suggestions. This work is partially supported by an RGC grant of the Hong Kong Government of the SAR of China under HKU7013/06P. L.M.L. acknowledges support from the Hong Kong Research Grants Council (grant no. 401807). The computations were performed on the Computational Grid of the Chinese University of Hong Kong and the High Performance Computing Cluster of the University of Hong Kong.

\section{REFERENCES}

Abdikamalov, E. B., Dimmelmeier, H., Rezzolla, L., \& Miller, J. C. 2009, MNRAS, 392, 52

Aksenov, A. G., Milgrom, M., \& Usov, V. V. 2003, MNRAS, 343, L69

Aksenov, A. G., Milgrom, M., \& Usov, V. V. 2005, ApJ, 632, 567

Alcock, C., Farhi, E., \& Olinto, A. 1986, ApJ, 310, 261

Alcock, C., \& Olinto, A. 1989, Phys. Rev. D, 39, 1233

Alekseev, E. N., Alekseeva, L. N., Krivosheina, I. V., \& Volchenko, V. I. 1988, Sov. Astron. Lett., 14, 41

Alford, M., Rajagopal, K., \& Wilczek, F. 1999, Nucl. Phys. B, 537, 443

Bailin, D., \& Love, A. 1984, Phys. Rep., 107, 325

Balantekin, A. B., \& Yuksel, H. 2005, New J. Phys., 7, 51

Baym, G., Bethe, H. A., \& Pethick, C. J. 1971, Nucl. Phys. A, 175, 221

Bejger, M., \& Haensel, P. 2004, A\&A, 420, 987

Bethe, H. A., \& Brown, G. E. 1995, ApJ, 445, L129

Bionta, R. M., Blewitt, G., Bratton, C. B., Caspere, D., \& Ciocio, A. 1987, Phys. Rev. Lett., 58, 1494B

Blaschke, D., Klahn, T., \& Voskresensky, D. N. 2000, ApJ, 533, 406

Bodmer, A. R. 1971, Phys. Rev. D, 4, 1601
Bombaci, I., Parenti, I., \& Vidana, I. 2004, ApJ, 614, 314

Bonanno, A., Urpin, V., \& Belvedere, G. 2005, A\&A, 440, 199

Caldwell, R. R., \& Friedman, J. R. 1991, Phys. Lett. B, 264, 143

Chen, A., Yu, T., \& Xu, R. 2007, ApJL, 668, 55

Cheng, K. S., Chau, W. Y., Zhang, J. L., \& Chau, H. F. 1992, ApJ, 396, 135

Cheng, K. S., \& Dai, Z. G. 1996, Phys. Rev. Lett., 77, 1210

Cheng, K. S., \& Dai, Z. G. 1998, Phys. Rev. Lett., 80, 18

Cheng, K. S., Dai, Z. G., \& Lu, T. 1998a, Int. J. Mod. Phys. D, 7, 139

Cheng, K. S., Dai, Z. G., Wei, D. M., \& Lu, T. 1998b, Science, 280, 407

Cheng, K. S., \& Harko, T. 2000, Phys. Rev. D, 30, 083001

Cheng, K. S., \& Harko, T. 2003, ApJ, 596, 451

Chmaj, T., Haensel, P., \& Slominski, W. 1991, Nucl. Phys. B (Proc. Suppl.), 24, 40

Chong, N., \& Cheng, K. S. 1993, ApJ, 417, 279

Christiansen, M. B., Glendenning, N. K., \& Schaffner-Bielich, J. 2000, Phys. Rev. C, 62, 025804

Chugunov, A. I. 2006, MNRAS, 371, 1659

Costantini, M. L., Ianni, A., Pagliaroli, G., \& Vissani, F. 2007, J. Cosmol. Astropart. Phys., JCAP05(2007)014

Costantini, M. L., Ianni, A., \& Vissani, F. 2004, Phys. Rev. D, 70, 043006

Dai, Z. G., \& Cheng, K. S. 1997, Phys. Lett. B, 401, 219

Dai, Z. G., Peng, Q. H., \& Lu, T. 1995, ApJ, 440, 815

DeGrand, T., Jaffe, R. L., Johnson, K., \& Kiskis, J. 1975, Phys. Rev. D, 12, 2060

Drago, A., Lavagno, A., \& Parenti, I. 2007, ApJ, 659, 1519

Duncan, R. C., Shapiro, S. L., \& Wasserman, I. 1983, ApJ, 267, 358

Fryer, C. L., Colgate, S. A., \& Pinto, P. A. 1999, ApJ, 511, 885

Gentile, N. A., Aufderheide, M. B., Mathews, G. J., Swesty, F. D., \& Fuller, G. M. 1993, ApJ, 414, 701

Glendenning, N. K. 2000, in Compact Stars, Nuclear Physics, Particle Physics and General Relativity, (New York: Springer)

Gondek-Rosinska, D., Gourgoulhon, E., \& Haensel, P. 2003, A\&A, 412, 777

Graves, G. J. M., et al. 2005, ApJ, 629, 944

Gudmundsson, E. H., Pethick, C. J., \& Epstein, R. I. 1983, ApJ, 272, 286

Haensel, P. 2003, in Final Stages of Stellar Evolution, ed. C. Hamenry \& J. M. Match (Les Ulis: EDP Sci.) 249

Haensel, P., Potekhin, A. Y., \& Yakovlev, D. G. 2007, in Neutron Stars. 1. Equation of State and Structure, (New York: Springer)

Haensel, P., \& Proszynski, M. 1982, ApJ, 258, 306

Haensel, P., \& Schaeffer, R. 1982, Nucl. Phys. A, 381, 519

Haensel, P., Zdunik, J. L., \& Schaeffer, R. 1986, A\&A, 160, 121

Halzen, F. 2006, Eur. Phys. J. C., 46, 669

Harko, T., \& Cheng, K. S. 2000, Phys. Lett. A, 266, 249

Harko, T., \& Cheng, K. S. 2002, A\&A, 385, 947

Harko, T., \& Cheng, K. S. 2005, ApJ, 622, 1033

Harko, T., \& Cheng, K. S. 2006, ApJ, 643, 318

Harko, T., Cheng, K. S., \& Tang, P. S. 2004, ApJ, 608, 945

Heinke, C. O., Jonker, P. G., Wijnands, R., \& Taam, R. E. 2007, ApJ, 660, 1424

Hirata, K. S., Kajita, T., Koshiba, M., Nakahata, M., \& Oyama, Y. 1987, Phys. Rev. Lett., 58, 1490

Horvath, J. E., \& Benvenuto, O. G. 1988, Phys. Lett. B, 213, 516

Iida, K., \& Sato, K. 1998, Phys. Rev. C, 58, 2538 
Itoh, N. 1970, Prog. Theor. Phys., 44, 291

Iwamoto, N. 1980, Phys. Rev. Lett., 44, 1637

Jaikumar, P., Gale, C., Page, D., \& Prakash, M. 2004, Phys. Rev. D, 70, 023004

Janka, H.-T. 1995, Astropart. Phys., 3, 377

Janka, H.-T. 2001, A\&A, 368, 527

Landau, L. D., \& Lifshitz, E. M. 1980, in Statistical Physics (Oxford: Pergamon)

Lin, L. M., Cheng, K. S., Chu, M. C., \& Suen, W. M. 2006, ApJ, 639, 382

Lugones, G., \& Benvenuto, O. G. 1995, Phys. Rev. D, 52, 1276

Lugones, G., Benvenuto, O. G., \& Vucetich, H. 1994, Phys. Rev. D, 50, 6100

Madsen, J. 1992, Phys. Rev. D, 46, 3290

Manchester, R. N. 2007, in in AIP Conf. Ser. 937, Supernova 1987A: 20 Years After, Supernovae and Gamma-Ray Bursters, ed. S. Immler, K. Weiler, \& R. McCray (Melville, NY: AIP), 134

Manchester, R. N., \& Peterson, B. A. 1996, ApJ, 456, L107

Maxwell, O. V. 1979, ApJ, 231, 201

Muto, T., \& Tatsumi, T. 1990, Progr. Theor. Phys., 83, 499

Muto, T., Tatsumi, T., \& Iwamoto, N. 1997, Aust. J. Phys., 50, 13

Muto, T., Tatsumi, T., \& Iwamoto, N. 2000a, Phys. Rev. D, 61, 063001

Muto, T., Tatsumi, T., \& Iwamoto, N. 2000b, Phys. Rev. D, 61, 083002

Ng, C. Y., Cheng, K. S., \& Chu, M. C. 2003, Astropart. Phys., 19, 171

Norsen, T. 2002, Phys. Rev. C, 65, 045805

Olinto, A. V. 1987, Phys. Lett. B, 192, 71

Paczynski, B., \& Haensel, P. 2005, MNRAS, 362, L4

Page, D., \& Usov, V. V. 2002, Phys. Rev. Lett., 89, 131101

Park, S., Zhekov, S. A., Burrows, D. N., Garmire, G. P., \& McCray, R. 2004, ApJ, 610,275

Percival, J. W., et al. 1995, ApJ, 446, 832

Rajagopal, K., \& Wilczek, F. 2001, Phys. Rev. Lett., 86, 3492

Satz, H. 1982, Phys. Lett. B, 113, 245

Sawyer, R. F. 1989, Phys. Rev. D, 39, 3804
Shapiro, S. L., \& Teukolsky, S. A. 1983, in Black Holes, White Dwarfs and Neutron Stars, (New York: Wiley)

Shen, H., Toki, H., Oyamatsu, K., \& Sumiyoshi, K. 1998, Nucl. Phys. A, 637 435

Shtykovskiy, P. E., Lutovinov, A. A., Gilfanov, M. R., \& Sunyaev, R. A. 2005, Astron. Lett., 31, 258

Steffens, F. M., Holtmann, H., \& Thomas, A. W. 1995, Phys. Lett. B, 358, 139

Svidzinsky, A. A. 2003, ApJ, 590, 386

Takahara, M., \& Sato, K. 1985a, ApJ, 335, 301

Takahara, M., \& Sato, K. 1985b, Phys. Lett. B, 156, 17

Takatsuka, T., \& Tamagaki, R. 1971, Prog. Theor. Phys., 46, 114

Tsuruta, S. 1998, Phys. Rep., 292, 1

Usov, V. V. 1998a, Phys. Rev. Lett., 80, 230

Usov, V. V. 1998b, Phys. Rev. Lett., 81, 4775

Usov, V. V. 2001, ApJ, 550, L179

Utrobin, V. P. 2007, in AIP Conf. Ser. 937, Supernova 1987 A: 20 Years After, Supernovae and Gamma-Ray Bursters, ed. S. Immler, K. Weiler, \& R. McCray (Melville, NY: AIP), 25

Vidana, I., Bombaci, I., \& Parenti, I. 2005, J. Phys. G: Nucl. Part. Phys., 31, S1165

Wang, G.-H., Fu, W.-J., \& Liu, Y.-X. 2007, Phys. Rev. C, 76, 065802

Wang, Q. D., \& Lu, T. 1984, Phys. Lett. B, 148, 211

Watts, A. L., \& Reddy, S. 2007, MNRAS, 379, L63, 2007

Wilson, J. R., \& Mayle, R. W. 1988, Phys. Rep., 163, 63

Witten, E. 1984, Phys. Rev. D, 30, 272

Yakovlev, D. G., \& Haensel, P. 2003, A\&A, 407, 259

Yakovlev, D. G., \& Pethick, C. J. 2004, ARA\&A, 42, 169

Yüksel, H., \& Beacom, J. F. 2007, Phys. Rev. D, 76, 083007

Zdunik, J. L., Bejger, M., Haensel, P., \& Gourgoulhon, E. 2006, A\&A, 450, 747

Zdunik, J. L., Haensel, P., \& Gourgoulhon, E. 2001, A\&A, 372, 535 